\title{
A CLOSEDNESS OF SET OF DIRICHLET SERIES SUMS
}

\section{A.S. KRIVOSHEYEV, O.A. KRIVOSHEYEVA}

\begin{abstract}
In the work we consider Dirichlet series. We study the problem of closedness for the set of the sums for such series in the space of functions holomorphic in a convex domain of a complex plane with a topology of uniform convergence on compact subsets. We obtain necessary and sufficient conditions under those each function in the closure of a linear span of exponents with positive indices is represented by a Dirichlet series. These conditions can be formulated only in terms of geometric characteristics of an index sequence and of the convex domain.
\end{abstract}

Keywords: exponential, convex domain, Dirichlet series, entire function, invariant subspace.

Mathematics Subject Classification: 41A05, 4130.

\section{INTRODUCTION}

Let $\Lambda=\left\{\lambda_{k}\right\}_{k=1}^{\infty}$ be an unbounded strictly increasing sequence of positive numbers. In the work we consider the Dirichlet series

$$
\sum_{k=1}^{\infty} d_{k} \exp \left(\lambda_{k} z\right) .
$$

It is known (see, for instance, [1, Ch. II, Sect. 1, Subsect. 4]) that under certain natural condition for the exponents $\lambda_{k}$, series (1) converges absolutely and uniformly on the compact sets in the half-plane $\{z \in \mathbb{C}: \operatorname{Re} z<\gamma\}$ to an analytic function and diverges in the half-plane $\{z \in \mathbb{C}: \operatorname{Re} z>\gamma\}$. The number $\gamma$ called convergence abscissa is calculated by a formula being an analogue for Cauchy-Hadamard formula for power series (see, for instance, [1, Ch. II, Sect. 1, Subsect. 4, Th. 2.1.2]). We also note the expansion of a function into the Dirichlet series is always unique (see, for instance, [1, Ch. II, Sect. 1, Subsect. 3]).

Let $D$ be a convex domain in $\mathbb{C}$ and $H(D)$ stand for the space of analytic in $D$ function with the topology of uniform convergence on compact subsets in $D$. The aim of this work is to find out the conditions under those the set of the sums of series (1), whose convergence half-planes contain domain $D$, is a closed subset in space $H(D)$.

This set contains a linear span of the system $\mathcal{E}=\left\{\exp \left(\lambda_{k} z\right)\right\}_{k=1}^{\infty}$ and it is a part of the subspace $W(\Lambda, D)$, which a closure of linear span $\mathcal{E}$ in $H(D)$. Subspace $W(\Lambda, D)$ is closed and invariant w.r.t. the differentiation operator. System $\mathcal{E}$ is the set of eigenfunctions for this operator in $W(\Lambda, D)$ and sequence $\Lambda$ is its spectrum. The definition of space $W(\Lambda, D)$ implies immediately that it admits the spectral synthesis, i.e., each of its functions is a limit of linear combinations of the eigenfunctions. It is easy to see that the closedness of sums of series (1) in $H(D)$ is equivalent to the fact that each function in $W(\Lambda, D)$ is represented by series (1) which converges uniformly on compact subsets of domain $D$. If $W(\Lambda, D)$ is nontrivial (i.e., system $\mathcal{E}$ is incomplete in $H(D)$ ) and the latter holds true, then one says that the fundamental principle holds true in subspace $W(\Lambda, D)$. The dual problem to that of

A.S. Krivosheyev, O.A. Krivosheyeva, A closedness of set of Dirichlet series sum.

(c) Krivosheyev A.S., Krivosheyeva O.A. 2013.

The work is supported by FTP (agreement no. 14.37.21.0358).

Submitted May 28, 2013. 
fundamental principle in a nontrivial closed invariant subspace of $H(D)$ admitting the spectral synthesis is the interpolation problem in the space of entire functions of exponential kind whose adjoint diagrams lie in domain $D$. The studies of both the problems made first independently have a rich history. The survey of main results obtained within these studies was presented in works [2] and [3]. A criterion of the fundamental principle (and for the interpolation as well) in arbitrary nontrivial closed invariant subspaces admitting spectral synthesis was obtain in works [3] and [4]. However, this criterion is formulated in terms of existence of certain special family of entire function vanishing at the points $\lambda_{k}, k \geq 1$, and obeying appropriate lower estimates. In the general case, especially for unbounded domains $D$, there is still an open question on the conditions for sequence $\Lambda$ and domain $D$ guaranteeing the existence of such family.

In the present work we obtain the complete solution for the problem on closedness of sums of series (1) for an aribtrary convex domain $D$ and, in particular, for the fundamental principle problems in the case of positive spectrum. In contrast to work [3], we employ simple geometric characteristics of sequence $\Lambda$.

In the second section we collect auxiliary results. In particular, we construct the aforementioned sequences of entire functions (Lemmata 7, 9) as well as an entire function in $W(\Lambda, \mathbb{C}$ ) which can not be represented by series (1) in any open subset (Lemma 3).

The main results of the work are given in the third section (Theorems 1-4). In particular, we prove that the set of the sums of the Dirichlet series converging in a given half-plane is closed if and only if $S_{\Lambda}>-\infty$. Quantity $S_{\Lambda}$ was introduced in work [3] (its definition is given in the second section). It is similar to the classical Bernstein condensation index (see, for instance, [1, Ch. II, Sect. 6, Subsect. 2]) but in contrast to the latter, it is effective for each complex sequence.

\section{Preliminary Results}

We shall make use of some results from the theory of entire functions of exponential type, i.e., of the functions $f$ satisfying the estimate: $\ln |f(z)| \leqslant A+B|z|, z \in \mathbb{C}$, where $A, B>0$ depend on $f$. As the upper and lower indicators of $f$ (subharmonic function $\ln |f|$ ), we call respectively the functions

$$
h_{f}(\lambda)=\varlimsup_{t \rightarrow \infty} \frac{\ln |f(t \lambda)|}{t}, \quad \underline{h}_{f}(\lambda)=\lim _{\delta \rightarrow 0} \lim _{t \rightarrow \infty} \frac{1}{\pi \delta^{2}} \int_{B(\lambda, \delta)} \frac{\ln |f(t z)|}{t} d x d y, \lambda \in \mathbb{C},
$$

where $z=x+i y$. By these definitions and Hartogs theorem on the upper limit of a family of subharmonic functions, it is easy to obtain the inequality $\underline{h}_{f}(\lambda) \leqslant h_{f}(\lambda), \lambda \in \mathbb{C}$. We say (cf. $[5$, Ch. II]) that $f$ has (completely) regular growth if

$$
h_{f}(\lambda)=\lim _{t \rightarrow \infty, t \notin E} \frac{\ln |f(t \lambda)|}{t}, \quad \lambda \in \mathbb{C},
$$

where $E$ is a set of zero relative measure on the ray $(0,+\infty)$, i.e., the Lebesgue measure of its intersection with the interval $(0, r)$ is infinitesimal w.r.t. $r$ as $r \rightarrow+\infty$. There is a series of other equivalent definitions of regular growth. Let us provide one of them. A function $f$ (cf. [6, Ch. 4, Def. 4.1]) is called function of regular growth if $\underline{h}_{f}(\lambda)=h_{f}(\lambda), \lambda \in \mathbb{C}$.

The upper indicator $h_{f}$ is a convex function positive homogeneous of order one which coincides with the support function of a convex compact set $K$ (more precisely, complex conjugate with $K$ compact set) called adjoint diagram of $f$ (see, for instance, [7, Ch. I, Sect. 5, Th. 5.4 (Pólya)]),

$$
h_{f}(\lambda)=H_{K}(\lambda)=\sup _{z \in K} \operatorname{Re}(\lambda z), \lambda \in \mathbb{C} .
$$

Let $\Lambda=\left\{\lambda_{k}\right\}_{k=1}^{\infty}$ be a sequence of complex numbers with the unique limiting point $\infty$. By the symbol $n\left(\varphi_{1}, \varphi_{2}, r, \Lambda\right)$ we denote the number of points $\lambda_{k}$ lying in the sector $\left\{\lambda=t e^{i \varphi}: \varphi \in\right.$ 
$\left.\left(\varphi_{1}, \varphi_{2}\right), t \in(0, r)\right\}$. We say (see [5, Ch. II, Sect. 1]) that $\Lambda$ has an angular density (as of order one) if for each $\varphi_{1}, \varphi_{2}$ except possible countable set there exists the limit

$$
\tau\left(\varphi_{1}, \varphi_{2}, \Lambda\right)=\lim _{r \rightarrow \infty} \frac{n\left(\varphi_{1}, \varphi_{2}, r, \Lambda\right)}{r} .
$$

Set $\Lambda$ is called well-distributed if it has an angular density and there exists

$$
\lim _{r \rightarrow \infty} \sum_{\left|\lambda_{k}\right|<r} \frac{1}{\lambda_{k}} .
$$

According to Theorem 4 in Chapter III in book [5], a function $f$ has a regular growth if and only if its zero set (counting multiplicities) is well-distributed. At that, if $K$ is the adjoint diagram for $f$, then except possible a countable number of values $\varphi_{1}, \varphi_{2}$, the identity

$$
\tau\left(\varphi_{1}, \varphi_{2}, \Lambda\right)=\frac{1}{2 \pi} s\left(\varphi_{1}, \varphi_{2}, K\right)
$$

holds true [5, Ch. II, Sect. 1, Formula (2.07)], where $s\left(\varphi_{1}, \varphi_{2}, K\right)$ is the length of the arc on boundary $\partial K$ between the support points $z\left(\varphi_{1}\right) \in \partial K$ and $z\left(\varphi_{2}\right) \in \partial K$ of respectively support lines $l\left(\varphi_{1}\right)=\left\{z: \operatorname{Re}\left(z e^{i \varphi_{1}}\right)=H_{K}\left(e^{i \varphi_{1}}\right)\right\}$ and $l\left(\varphi_{2}\right)=\left\{z: \operatorname{Re}\left(z e^{i \varphi_{2}}\right)=H_{K}\left(e^{i \varphi_{2}}\right)\right\}$. Except possible countable set of values $\varphi$ (associated with straight parts of the boundary), support line $l(\varphi)$ has the only support point $z(\varphi)$. Among two arcs connecting points $z\left(\varphi_{1}\right)$ and $z\left(\varphi_{2}\right)$, we choose one so that each its point is the support one of some straight line $l(\varphi)$ (depending on it) with value of $\varphi$ in the segment $\left[\varphi_{1}, \varphi_{2}\right]$. In the case $K$ is the segment of length $\tau$ (and only in this case), the length of the $\operatorname{arc} s\left(\varphi_{1}, \varphi_{2}, K\right)$, where $\varphi_{1}$ and $\varphi_{2}$ are not equal to two opposite numbers $\varphi_{0}$ and $-\varphi_{0}$, can take just one of three possible values: 0 if the interval $\left(\varphi_{1}, \varphi_{2}\right)$ contains none of these numbers, $\tau$ if it contains just one of them, and $2 \tau$ if $-\varphi_{0}, \varphi_{0} \in\left(\varphi_{1}, \varphi_{2}\right)$.

Let $D$ be a convex domain in $\mathbb{C}$ and $H^{*}(D)$ be the strongly adjoint space for $H(D)$. The Laplace transform $f(\lambda)=\nu(\exp (\lambda z))$ makes an isomorphism (see, for instance, [8, Ch. III, Sect. 12, Th. 12.3]) between $H^{*}(D)$ and space $P_{D}$ consisting of entire functions of exponential type whose adjoint diagrams lie in domain $D$. By Hahn-Banach theorem, the incompleteness of the system $\mathcal{E}=\left\{\exp \left(\lambda_{k} z\right)\right\}_{k=1}^{\infty}$ in $H(D)$ (i.e., non-triviality of $W(\Lambda, D)$ ) is equivalent to the existence of a non-zero linear continuous functional $\nu \in H^{*}(D)$ vanishing at the elements of the system. Thus, the incompleteness of $\mathcal{E}$ is equivalent to the existence of the function $f \in P_{D}$ vanishing at the points $\lambda_{k}, k=1,2, \ldots$

Let $\Lambda=\left\{\lambda_{k}\right\}_{k=1}^{\infty}$ be an unbounded strictly increasing sequence of positive numbers $n(r, \Lambda)$ and denotes the numbers of its terms lying in the semi-interval $(0, r]$. We sat that $\Lambda$ has density $\tau(\Lambda)$ (is measurable) if there exists a limit

$$
\tau(\Lambda)=\lim _{r \rightarrow \infty} \frac{n(r, \Lambda)}{r} .
$$

As maximal density of sequence $\Lambda$, we call the quantity

$$
\tau_{0}(\Lambda)=\varlimsup_{\delta \rightarrow 0} \varlimsup_{r \rightarrow \infty} \frac{n(r, \Lambda)-n((1-\delta) r, \Lambda)}{\delta r} .
$$

We note that in accordance with Lemma in Section E3 of Chapter VI in book [9], the upper limit as $\delta \rightarrow 0$ in the definition of $\tau_{0}(\Lambda)$ can be replaced by the limit since it always exists. We let

$$
\underline{\tau}(\Lambda)=\lim _{r \rightarrow \infty} \frac{n(r, \Lambda)}{r}, \quad \bar{\tau}(\Lambda)=\varlimsup_{r \rightarrow \infty} \frac{n(r, \Lambda)}{r} .
$$

Quantities $\underline{\tau}(\Lambda)$ and $\bar{\tau}(\Lambda)$ are called respectively lower and upper density for sequence $\Lambda$. The latter is measurable if and only if $\underline{\tau}(\Lambda)=\bar{\tau}(\Lambda)$. It is easy to see that inequalities

$$
\underline{\tau}(\Lambda) \leqslant \bar{\tau}(\Lambda) \leqslant \tau_{0}(\Lambda)
$$


hold true. The former follows immediately from the definitions. For the case $\bar{\tau}(\Lambda)<+\infty$, the latter is implied by the relations

$$
\begin{gathered}
\varlimsup_{r \rightarrow \infty} \frac{n(r, \Lambda)-n((1-\delta) r, \Lambda)}{\delta r} \geq \varlimsup_{r \rightarrow \infty} \frac{n(r, \Lambda)}{\delta r}-\varlimsup_{r \rightarrow \infty} \frac{n((1-\delta) r, \Lambda)}{\delta r}= \\
=\frac{\bar{\tau}(\Lambda)}{\delta}-(1-\delta) \varlimsup_{r \rightarrow \infty} \frac{n((1-\delta) r, \Lambda)}{(1-\delta) \delta r}=\frac{\bar{\tau}(\Lambda)}{\delta}-(1-\delta) \frac{\bar{\tau}(\Lambda)}{\delta}=\bar{\tau}(\Lambda), \delta>0 .
\end{gathered}
$$

If $\bar{\tau}(\Lambda)=+\infty$, then one can easily select a subsequence $\Lambda^{\prime}$ in sequence $\Lambda$ with an arbitrarily large finite upper density. Then by the proven we obtain: $\bar{\tau}\left(\Lambda^{\prime}\right) \leqslant \tau_{0}\left(\Lambda^{\prime}\right) \leqslant \tau_{0}(\Lambda)$. Therefore, $\tau_{0}(\Lambda)=+\infty$, i.e., inequality (3) is valid in this case as well. Similar arguments show that in the case when the sequence has density $\tau(\Lambda)$, the identity $\tau_{0}(\Lambda)=\tau(\Lambda)$ holds true. In the general case, the second inequality in (3) can be strict. Indeed, consider the following example. Let $\Lambda=\cup_{m=1}^{\infty} \Lambda_{m}$, where $\Lambda_{m}=\left\{\lambda_{k}, k(m) \leqslant k<k(m+1)\right\}, \lambda_{k(m)+j}=10^{m}+j$, $0 \leqslant j<k(m+1)-k(m), m=1,2, \ldots$, and $k(1)=1, k(m+1)-k(m)=10^{m-1}$ as $m>1$. By direct calculations one can easily get the relations $\bar{\tau}(\Lambda) \leqslant 1 / 9, \tau_{0}(\Lambda)=1$.

Let $\Lambda=\left\{\lambda_{k}\right\}_{k=1}^{\infty}$ be a complex sequence. Following work [3], we let

$$
q_{\Lambda}^{j}(z, \delta)=\prod_{\lambda_{k} \in B\left(\lambda_{j}, \delta\left|\lambda_{j}\right|\right), k \neq j}\left(\frac{z-\lambda_{k}}{3 \delta\left|\lambda_{k}\right|}\right) .
$$

Here $B(w, r)$ is an open circle centered at $w$ with radius $r$. The absolute value of the function $q_{\Lambda}^{j}(z, \delta)$ can be interpreted as a measure of accumulation of the points $\lambda_{k} \in B\left(\lambda_{j}, \delta\left|\lambda_{j}\right|\right), k \neq j$, at $z$. In the case of absence of such points, we assume that $q_{\Lambda}^{j}(z, \delta) \equiv 1$. We note that the absolute value of each of multipliers in the definition of $q_{\Lambda}^{j}$, in the circle $B\left(\lambda_{j}, \delta\left|\lambda_{j}\right|\right)$ is estimated from above by the quantity $2(3(1-\delta))^{-1}$ (for $\delta \in(0,1)$ ), i.e., as $\delta \in(0,1 / 3)$, it does not exceeds one. Moreover, if $\delta_{1} \leqslant \delta_{2}$, the number of multipliers in the definition of $q_{\Lambda}^{j}\left(z, \delta_{1}\right)$ does not exceeds the number of multipliers in the definition of $q_{\Lambda}^{j}\left(z, \delta_{2}\right)$ and each of the multipliers for $q_{\Lambda}^{j}\left(z, \delta_{1}\right)$ is not less in absolute value than the corresponding multiplier for $q_{\Lambda}^{j}\left(z, \delta_{2}\right)$. Thus, if $0<\delta_{1} \leqslant \delta_{2}<1 / 3$, then $\left|q_{\Lambda}^{j}\left(z, \delta_{1}\right)\right| \geq\left|q_{\Lambda}^{j}\left(z, \delta_{2}\right)\right|, z \in B\left(\lambda_{j}, \delta_{2}\left|\lambda_{j}\right|\right)$. We let $S_{\Lambda}=0$ if $\Lambda$ consists of a finite number of elements and

$$
S_{\Lambda}=\lim _{\delta \rightarrow 0} \lim _{k \rightarrow \infty} \frac{\ln \left|q_{\Lambda}^{k}\left(\lambda_{k}, \delta\right)\right|}{\left|\lambda_{k}\right|}
$$

otherwise. This definition is well-defined, since according to the latter inequality, the limit w.r.t. $\delta$ always exists. By the said above, $S_{\Lambda} \leqslant 0$. We note that the coefficient 3 in the definition of $q_{\Lambda}^{j}$ is chosen just for convenience (cf. [3, Rem. 1 to Th. 5.1]). It ensures the non-positivity for quantity $S_{\Lambda}$. It is close by the meaning with the classical Bernstein condensation index, but it is effective for each complex sequence, not only for a measurable positive sequence and a complex sequence of zero density. Together with $S_{\Lambda}$, we introduce one more quantity

$$
\tilde{S}_{\Lambda}=\lim _{\delta \rightarrow 0} \lim _{k \rightarrow \infty} \frac{\ln \left|q_{\Lambda}^{k}\left(\lambda_{k}, \delta\right)\right|}{\delta\left|\lambda_{k}\right|} .
$$

As for $S_{\Lambda}$, the inequality $\tilde{S}_{\Lambda} \leqslant 0$ is valid. If $\tilde{S}_{\Lambda}$ is finite, then, obviously, $S_{\Lambda}=0$. As an example we consider the sequence of positive numbers $\Lambda=\left\{\lambda_{k}\right\}_{k=1}^{\infty}$ such that $\lambda_{k+1}-\lambda_{k} \geq h>0$, $k=1,2, \ldots$ Taking into consideration the inequality $n ! \geq(n / 3)^{n}$, we have

$$
\left|q_{\Lambda}^{k}\left(\lambda_{k}, \delta\right)\right| \geq \prod_{\lambda_{m} \in B\left(\lambda_{k}, \delta \lambda_{k}\right), m \neq k}\left|\frac{\lambda_{m}-\lambda_{k}}{3 \delta \lambda_{m}}\right| \geq \frac{\left(m(k, \delta) ! h^{m(k, \delta)}\right)^{2}}{\left(3 \delta(1+\delta) \lambda_{k}\right)^{2 m(k, \delta)}} \geq \frac{(m(k, \delta) h)^{2 m(k, \delta)}}{\left(9 \delta(1+\delta) \lambda_{k}\right)^{2 m(k, \delta)}},
$$

where $m(k, \delta)$ is the maximal integer satisfying inequality $m(k, \delta) h<\delta \lambda_{k}$. Therefore,

$$
\tilde{S}_{\Lambda} \geq \lim _{\delta \rightarrow 0} \lim _{k \rightarrow \infty} \frac{2 m(k, \delta) \ln \left(m(k, \delta) h / 9 \delta(1+\delta) \lambda_{k}\right)}{\delta \lambda_{k}} \geq-\frac{2 \ln 9}{h} .
$$


Lemma 1. Let $\Lambda=\left\{\lambda_{k}\right\}_{k=1}^{\infty}$ be an unbounded strictly increasing sequence of positive numbers. Suppose that $\tilde{S}_{\Lambda}>-\infty$. Then the maximal density $\tau_{0}(\Lambda)$ is finite.

Proof. Let $\delta \in(0,1)$. Employing the definition of $q_{\Lambda}^{j}$, we obtain

$$
\begin{gathered}
\ln \left|q_{\Lambda}^{j}\left(\lambda_{j}, \delta\right)\right|=\ln \left|\prod_{\lambda_{k} \in B\left(\lambda_{j}, \delta \lambda_{j}\right), k \neq j}\left(\frac{\lambda_{j}-\lambda_{k}}{3 \delta \lambda_{k}}\right)\right| \leqslant \ln \left|\prod_{\lambda_{k} \in B\left(\lambda_{j}, \delta \lambda_{j}\right), k \neq j}\left(\frac{\delta \lambda_{j}}{3 \delta \lambda_{k}}\right)\right| \leqslant \\
\leqslant \ln \left(\frac{\lambda_{j}}{3(1-\delta) \lambda_{j}}\right)^{(m(j, \delta)-1)}=-(m(j, \delta)-1) \ln (3(1-\delta)),
\end{gathered}
$$

where $m(j, \delta)$ is the number of points $\lambda_{k}$ in the circle $B\left(\lambda_{j}, \delta \lambda_{j}\right)$, i.e., $m(j, \delta)=n\left((1+\delta) \lambda_{j}, \Lambda\right)-$ $-n\left((1-\delta) \lambda_{j}, \Lambda\right)$ if the point $(1+\delta) \lambda_{j}$ does not belong to $\Lambda$, and the number $m(j, \delta)$ is less by one otherwise. Therefore, we have

$$
\begin{aligned}
& \tilde{S}_{\Lambda}=\lim _{\delta \rightarrow 0} \lim _{k \rightarrow \infty} \frac{\ln \left|q_{\Lambda}^{k}\left(\lambda_{k}, \delta\right)\right|}{\delta \lambda_{k}} \leqslant \lim _{\delta \rightarrow 0} \lim _{j \rightarrow \infty} \frac{-\ln (3(1-\delta)) m(j, \delta)}{\delta \lambda_{j}}= \\
& =-\varlimsup_{\delta \rightarrow 0} \ln (3(1-\delta)) \varlimsup_{j \rightarrow \infty} \frac{m(j, \delta)}{\delta \lambda_{j}}=-\ln 3 \varlimsup_{\delta \rightarrow 0} \varlimsup_{j \rightarrow \infty} \frac{n\left((1+\delta)\left|\lambda_{j}\right|, \Lambda\right)-n\left((1-\delta)\left|\lambda_{j}\right|, \Lambda\right)}{\delta \lambda_{j}} .
\end{aligned}
$$

Let us show that the double upper limit in the latter identity is estimated from below by the quantity $\tau_{0}(\Lambda)$. If $\tau_{0}(\Lambda)=0$, it is obvious. For each $\delta \in(0,1)$, by $r_{k}(\delta), k=1,2, \ldots$ we denote the sequence implementing the upper limit as $r \rightarrow \infty$ in the definition of the maximal density. Let $\tau_{0}(\Lambda)>0$. Then we can assume that each semi-interval $\left((1-\delta) r_{k}(\delta), r_{k}(\delta)\right]$ contains some nonzero number of sequence $\Lambda$. We choose arbitrarily one of them and by $j(k, \delta)$ we indicate its index. Since $\lambda_{j(k, \delta)} \leqslant r_{k}(\delta) \leqslant \lambda_{j(k, \delta)} /(1-\delta)$, it is easy to observe that the inclusion $\left((1-\delta) r_{k}(\delta), r_{k}(\delta)\right] \subset\left((1-\tilde{\delta}) \lambda_{j(k, \delta)},(1+\tilde{\delta}) \lambda_{j(k, \delta)}\right]$ holds true, where $\tilde{\delta}=\delta /(1-\delta)$. Thus,

$$
\begin{gathered}
\tau_{0}(\Lambda)=\varlimsup_{\delta \rightarrow 0} \varlimsup_{r \rightarrow \infty} \frac{n(r, \Lambda)-n((1-\delta) r, \Lambda)}{\delta r}=\varlimsup_{\delta \rightarrow 0} \lim _{k \rightarrow \infty} \frac{n\left(r_{k}(\delta), \Lambda\right)-n\left((1-\delta) r_{k}(\delta), \Lambda\right)}{\delta r_{k}(\delta)} \leqslant \\
\leqslant \varlimsup_{\delta \rightarrow 0} \frac{\tilde{\delta}}{\varlimsup_{j \rightarrow \infty}} \frac{n\left((1+\tilde{\delta}) \lambda_{j}, \Lambda\right)-n\left((1-\tilde{\delta}) \lambda_{j}, \Lambda\right)}{\tilde{\delta} \lambda_{j}}= \\
=\varlimsup_{\tilde{\delta} \rightarrow 0} \varlimsup_{r \rightarrow \infty} \frac{n\left((1+\tilde{\delta}) \lambda_{j}, \Lambda\right)-n\left((1-\tilde{\delta}) \lambda_{j}, \Lambda\right)}{\tilde{\delta} \lambda_{j}} .
\end{gathered}
$$

Hence, in view of the above arguments, we obtain $\tilde{S}_{\Lambda} \leqslant-\ln 3 \tau_{0}(\Lambda)$. It implies the required statement. The proof is complete.

Lemma 2. Let $\Lambda=\left\{\lambda_{k}\right\}_{k=1}^{\infty}$ be an unbounded strictly increasing sequence of positive numbers. If $S_{\Lambda}>-\infty$, then $\bar{\tau}(\Lambda)<+\infty$

Proof. Suppose $\bar{\tau}(\Lambda)=+\infty$. Then for each $A>0$ there exists a subsequence $\Lambda(A)$ of sequence $\Lambda$ such that the upper density $\bar{\tau}(\Lambda(A))$ is finite and greater than $A$. We fix $\delta \in(0,1)$ and $A>0$. We have

$$
\begin{gathered}
\bar{\tau}(\Lambda(A))=\varlimsup_{r \rightarrow \infty} \frac{n(r, \Lambda(A))}{r} \leqslant \varlimsup_{r \rightarrow \infty} \frac{n(r, \Lambda(A))-n((1-\delta) r, \Lambda(A))}{r}+\varlimsup_{r \rightarrow \infty} \frac{n((1-\delta) r, \Lambda(A))}{r}= \\
=\varlimsup_{r \rightarrow \infty} \frac{n(r, \Lambda(A))-n((1-\delta) r, \Lambda(A))}{r}+(1-\delta) \bar{\tau}(\Lambda(A)) .
\end{gathered}
$$

It follows that for each $A>0$,

$$
\delta A \leqslant \delta \bar{\tau}(\Lambda(A)) \leqslant \varlimsup_{r \rightarrow \infty} \frac{n(r, \Lambda(A))-n((1-\delta) r, \Lambda(A))}{r} \leqslant \varlimsup_{r \rightarrow \infty} \frac{n(r, \Lambda)-n((1-\delta) r, \Lambda)}{r} .
$$


Hence,

$$
\varlimsup_{r \rightarrow \infty} \frac{n(r, \Lambda)-n((1-\delta) r, \Lambda)}{r}=+\infty, \quad \delta \in(0,1) .
$$

Let $\delta \in(0,1 / 2)$. We choose a sequence $r_{j} \rightarrow+\infty$ such that

$$
\lim _{j \rightarrow \infty} \frac{n\left(r_{j}, \Lambda\right)-n\left((1-\delta) r_{j}, \Lambda\right)}{r_{j}}=+\infty .
$$

We can assume that for each $j \geq 1$, the semi-interval $\left((1-\delta) r_{j}, r_{j}\right]$ contains some points of sequence $\Lambda$. Let $\lambda_{k_{j}}$ be one of such points. Then the inclusion $\left((1-\delta) r_{j}, r_{j}\right] \subset\left((1-2 \delta) \lambda_{k_{j}},(1+2 \delta) \lambda_{k_{j}}\right)$ holds true. This is why

$$
\lim _{j \rightarrow \infty} \frac{m\left(k_{j}, 2 \delta\right)}{\lambda_{k_{j}}}=+\infty
$$

where $m\left(k_{j}, 2 \delta\right)$ is defined in Lemma 1 . As in this lemma, we obtain

$$
\ln \left|q_{\Lambda}^{k_{j}}\left(\lambda_{k_{j}}, 2 \delta\right)\right| \leqslant-\left(m\left(k_{j}, 2 \delta\right)-1\right) \ln (3(1-2 \delta)) .
$$

Therefore, we have

$$
\begin{aligned}
& S_{\Lambda}=\lim _{\delta \rightarrow 0} \frac{\lim _{k \rightarrow \infty}}{\ln \left|q_{\Lambda}^{k}\left(\lambda_{k}, \delta\right)\right|} \lambda_{k} \leqslant \lim _{\delta \rightarrow 0} \lim _{j \rightarrow \infty} \frac{\ln \left|q_{\Lambda}^{k_{j}}\left(\lambda_{k_{j}}, 2 \delta\right)\right|}{\lambda_{k_{j}}} \leqslant \\
& \leqslant \lim _{\delta \rightarrow 0} \ln (3(1-2 \delta)) \lim _{j \rightarrow \infty} \frac{-\left(m\left(k_{j}, 2 \delta\right)-1\right)}{\lambda_{k_{j}}}=-\ln 3 \lim _{\delta \rightarrow 0} \varlimsup_{j \rightarrow \infty} \frac{\left(m\left(k_{j}, 2 \delta\right)-1\right)}{\lambda_{k_{j}}}=-\infty .
\end{aligned}
$$

It contradicts the assumption. Thus, $\bar{\tau}(\Lambda)<+\infty$. The proof is complete.

By $S(z, r)$ we denote the circumference centered at $z$ of radius $r>0$. The proof of the next two statements is based on the ideas of the proof of Theorem 3.1 in work [10].

Lemma 3. Let $\Lambda=\left\{\lambda_{k}\right\}_{k=1}^{\infty}$ be an unbounded strictly increasing sequence of positive numbers such that $S_{\Lambda}=-\infty$. Then there exists an entire function $g \in W(\Lambda, \mathbb{C})$ which does not expand into a Dirichlet series w.r.t. the system $\mathcal{E}=\left\{\exp \left(\lambda_{k} z\right)\right\}_{k=1}^{\infty}$ on any open subset in the plane.

Proof. Suppose first that $\bar{\tau}(\Lambda)<+\infty$. By assumption, $S_{\Lambda}=-\infty$. Hence, there exist a sequence of positive numbers $\left\{\delta_{p}\right\}$ and a subsequence $\left\{\lambda_{k(p)}\right\}$ such that $\delta_{p} \rightarrow 0$ as $p \rightarrow \infty$ and

$$
\lim _{p \rightarrow 0} \frac{\ln \left|q_{\Lambda}^{k(p)}\left(\lambda_{k(p)}, \delta_{p}\right)\right|}{\lambda_{k(p)}}=-\infty
$$

We can assume that

$$
\lambda_{k(p+1)} \geq 2 \lambda_{k(p)}, \quad \delta_{p}<1 / 4, \quad p \geq 1
$$

Consider the functions

$$
g_{p}(z)=\frac{1}{2 \pi i} \int_{S\left(\lambda_{k(p)}, 5 \delta_{p} \lambda_{k(p)}\right)} \frac{\exp (\lambda z) d \lambda}{\left(\lambda-\lambda_{k(p)}\right) q_{\Lambda}^{k(p)}\left(\lambda, \delta_{p}\right)}, \quad p=1,2, \ldots
$$

Let us find the upper estimate for $\left|g_{p}\right|$. We have

$$
\begin{gathered}
\left|q_{\Lambda}^{k(p)}\left(\lambda, \delta_{p}\right)\right|=\left|\prod_{\lambda_{k} \in B\left(\lambda_{k(p)}, \delta_{p} \lambda_{k(p)}\right), k \neq k(p)} \frac{\lambda-\lambda_{k}}{3 \delta_{p} \lambda_{k}}\right| \geq \\
\geq\left(\frac{4 \delta_{p} \lambda_{k(p)}}{3 \delta_{p}\left(1+\delta_{p}\right) \lambda_{k(p)}}\right)^{m\left(k(p), \delta_{p}\right)} \geq 1, \quad \lambda \in S\left(\lambda_{k(p)}, 5 \delta_{p} \lambda_{k(p)}\right),
\end{gathered}
$$


where $m\left(k(p), \delta_{p}\right)$ is determined in Lemma 1 . Thus, the inequalities

$$
\begin{gathered}
\left|g_{p}(z)\right|=\left|\frac{1}{2 \pi i} \int_{S\left(\lambda_{k(p)}, 5 \delta_{p} \lambda_{k(p)}\right)} \frac{\exp (\lambda z) d \lambda}{\left(\lambda-\lambda_{k(p)}\right) q_{\Lambda}^{k(p)}\left(\lambda, \delta_{p}\right)}\right| \leqslant \\
\leqslant 5 \lambda_{k(p)} \delta_{p} \sup _{\lambda \in S\left(\lambda_{k(p)}, 5 \delta_{p} \lambda_{k(p)}\right)}\left|\frac{\exp (\lambda z)}{\left(\lambda-\lambda_{k(p)}\right)}\right| \leqslant \sup _{\lambda \in S\left(\lambda_{k(p)}, 5 \delta_{p} \lambda_{k(p)}\right)}|\exp (\lambda z)| \leqslant \\
\leqslant \exp \left(\operatorname{Re}\left(\lambda_{k(p)} z\right)+5 \delta_{p} \lambda_{k(p)}|z|\right), \quad z \in \mathbb{C},
\end{gathered}
$$

hold true.

Consider the function

$$
g(z)=\sum_{p=1}^{\infty} c_{p} g_{p}(z)
$$

where $c_{p}=\sqrt{\left|q_{\Lambda}^{k(p)}\left(\lambda_{k(p)}, \delta_{p}\right)\right|}, p \geq 1$. Let us show that this series converges uniformly on each compact subset of the plane. Let $R>0$. By (4), there exists a number $p_{0}$ such that $\left|c_{p}\right| \leqslant \exp \left(-2 R \lambda_{k(p)}\right), p \geq p_{0}$. Then in view of (6) we have

$$
\sum_{p=1}^{\infty}\left|c_{p}\right| \max _{|z| \leqslant R}\left|g_{p}(z)\right| \leqslant A+\sum_{p=p_{0}}^{\infty} \exp \left(-2 R \lambda_{k(p)}+R \lambda_{k(p)}+5 \delta_{p} R \lambda_{k(p)}\right)<+\infty .
$$

This estimate holds true thanks to inequalities $\lambda_{k(p+1)} \geq 2 \lambda_{k(p)}, p \geq 1$ and that $\delta_{p} \rightarrow 0$ as $p \rightarrow \infty$.

Thus, function $g(z)$ is entire and belongs to space $W(\Lambda, \mathbb{C})$. Suppose it is represented by series (1) on some open subset $U \subset \mathbb{C}$ containing a point $z_{0}$. Then by Abel theorem for Diriclhet series (see, for instance [1, Ch. II, Sect. 1, Subsect. 2], series (1) converges uniformly on compact subsets of the half-plane $\Pi=\left\{z \in \mathbb{C}: \operatorname{Re} z<\operatorname{Re} z_{0}\right\}$. On open subset $\Pi \cap U$ its sum is equal to $g(z)$. This is why it converges to $g(z)$ in the whole half-plane $\Pi$. Since upper density $\bar{\tau}(\Lambda)$ is finite, there exists (see, for instance, [1, Ch. IV, Sect. 1, Subsect. 1]) the biorthogonal to $\mathcal{E}$ sequence of functionals $\left\{\nu_{k}\right\} \subset H^{*}(\Pi) \subset H^{*}(\mathbb{C})$, i.e., $\nu_{k}\left(\exp \left(\lambda_{k} z\right)\right)=1$, $k \geq 1$, and $\nu_{k}\left(\exp \left(\lambda_{j} z\right)\right)=0$, if $k \neq j$. Since series (1) in the topology of space $H(\Pi)$, the identities

are valid.

$$
\nu_{k}(g)=d_{k}, \quad k \geq 1
$$

Employing the residua and the definition of function $g_{p}$, we obtain

$$
g_{p}(z)=b_{k(p)} \exp \left(\lambda_{k(p)} z\right)+\sum_{\lambda_{k} \in\left(\left(1-\delta_{p}\right) \lambda_{k(p)},\left(1+\delta_{p}\right) \lambda_{k(p)}\right), k \neq k(p)} b_{k} \exp \left(\lambda_{k} z\right),
$$

where $b_{k(p)}=\left(q_{\Lambda}^{k(p)}\left(\lambda_{k(p)}, \delta_{p}\right)\right)^{-1}, p \geq 1$. By (5), the intervals $\left(\left(1-\delta_{p}\right) \lambda_{k(p)},\left(1+\delta_{p}\right) \lambda_{k(p)}\right)$ are mutually disjoint. Then, taking into consideration the convergence of series (7) in the topology of space $H(\mathbb{C})$ and identities (8), we obtain

$$
\left|d_{k(p)}\right|=\left|\nu_{k(p)}(g)\right|=\left|c_{p} b_{k(p)}\right|=\left(\sqrt{\left|q_{\Lambda}^{k(p)}\left(\lambda_{k(p)}, \delta_{p}\right)\right|}\right)^{-1}, \quad p \geq 1 .
$$

It follows from (4) that for each $z \in \mathbb{C}$

$$
\left|d_{k(p)}\right| \exp \left(\operatorname{Re}\left(\lambda_{k(p)} z\right)\right) \rightarrow+\infty, \quad p \rightarrow \infty .
$$

It contradicts to the convergence of series (1) in half-plane $\Pi$. Thus, function $g \in W(\Lambda, \mathbb{C})$ does not expand into the series w.r.t. system $\mathcal{E}$ on any open subset of the plane.

It remains to consider the situation when $\bar{\tau}(\Lambda)=+\infty$. In this case there exists no (see, for instance, [1, Ch. I, Sect. 1, Th. 1.1.2]) entire function of exponential type $f \in P_{\mathbb{C}}$ vanishing at 
all points of $\lambda_{k}$. Therefore, system $\mathcal{E}$ is complete in $H(\mathbb{C})$, i.e., $W(\Lambda, \mathbb{C})=H(\mathbb{C})$. Let $\lambda_{0}>0$ differ from the points $\lambda_{k}, k \geq 1$. Suppose the function $\exp \left(\lambda_{0} z\right) \in W(\Lambda, \mathbb{C})$ is represented by series (1) on some open subset of the complex plane. As above, this representation can be extended on some half-plane. Then in this half-plane, the identity

$$
0=\sum_{k=0}^{\infty} d_{k} \exp \left(\lambda_{k} z\right)
$$

holds true, where $d_{0}=-1$. In the beginning of the work we mentioned that the representation by the Dirichlet series is always unique. Thus, the relations $d_{k}=0, k=0,1, \ldots$ hold true. We obtain the contradiction. The proof is complete.

Corollary. Let $D$ be a convex domain in $\mathbb{C}, \Lambda=\left\{\lambda_{k}\right\}_{k=1}^{\infty}$ be an unbounded strictly increasing sequence of positive numbers. Suppose the set of the sums of series (1) converging in domain $D$ is closed in space $H(D)$. Then $S_{\Lambda}>-\infty$ and $\bar{\tau}(\Lambda)<+\infty$.

Proof. If $S_{\Lambda}=-\infty$, by Lemma 3, there exists a function $g \in W(\Lambda, \mathbb{C}) \subset W(\Lambda, D)$ which does not expand into series (1) in domain $D$. It contradicts the assumption. Thus, $S_{\Lambda}>-\infty$. Then by Lemma $2, \bar{\tau}(\Lambda)<+\infty$. The proof is complete.

Lemma 4. Let $\Lambda=\left\{\lambda_{k}\right\}_{k=1}^{\infty}$ be an unbounded strictly increasing sequence of positive numbers. Suppose $\tilde{S}_{\Lambda}=-\infty$. Then for each $\tau>0$, there exists $\delta>0$ and function $g \in W(\Lambda, G)$, where $G=(\{z: \operatorname{Re} z<\tau \delta\} \cap B(0, \tau)) \cup\{z: \operatorname{Re} z<0\}$ is represented by series (1) with the convergence abscissa $\gamma=0$.

Proof. Let

$$
g(z)=\sum_{p=1}^{\infty} c_{p} g_{p}(z)
$$

where $c_{p}=\exp \left(-6 \tau \delta \mu_{m(p)}\right)$,

$$
g_{p}(z)=\frac{1}{2 \pi i} \int_{S\left(\mu_{m(p)}, 5 \delta \mu_{m(p)}\right)} \frac{\exp (\lambda z) d \lambda}{a_{p}\left(\lambda-\mu_{m(p)}\right) q_{\tilde{\Lambda}}^{m(p)}(\lambda, \delta)}, \quad p=1,2, \ldots,
$$

and the sequence $\tilde{\Lambda}=\left\{\mu_{m}\right\}_{m=1}^{\infty}$ is a part of $\Lambda$. We shall choose numbers $a_{p} \geq 1$ later. Now we determine number $\delta$, construct $\tilde{\Lambda}$, and select $m(p)$. In order to do it, we first of all observe that according to the assumption, $\tilde{S}_{\Lambda}=-\infty$. This is why the exist $\delta \in(0,1 / 4)$ and a subsequence $\left\{\lambda_{k(p)}\right\}_{p=1}^{\infty}$ of sequence $\Lambda$ obeying condition

$$
\ln \left|q_{\Lambda}^{k(p)}\left(\lambda_{k(p)}, \delta\right)\right| \leqslant-6 \tau \delta \lambda_{k(p)}, \quad p=1,2, \ldots
$$

At that, we can assume that

$$
\lambda_{k(p+1)} \geq 2 \lambda_{k(p)}, \quad p=1,2, \ldots
$$

We shall seek sequence $\tilde{\Lambda} \subset \Lambda$ as the union $\cup_{p=1}^{\infty} \Lambda_{p}$. Fix $p=1,2, \ldots$. If $n\left((1+\delta) \lambda_{k(p)}, \Lambda\right)-$ $n\left((1-\delta) \lambda_{k(p)}, \Lambda\right)-1<12 \tau \delta \lambda_{k(p)}+1$, as $\Lambda_{p}$, we take the set of all points in sequence $\Lambda$ lying in the circle $B\left(\lambda_{k(p)}, \delta \lambda_{k(p)}\right)$. Otherwise we put the point $\lambda_{k(p)}$ into $\Lambda_{p}$ and as many points in $\Lambda$ lying in the circle $B\left(\lambda_{k(p)}, \delta \lambda_{k(p)}\right)$ as we need the number of points $l(p)$ of set $\Lambda_{p}$ to satisfy the estimates

$$
12 \tau \delta \lambda_{k(p)} \leqslant l(p)-1<12 \tau \delta \lambda_{k(p)}+1
$$

We note that by (11) and the choice of number $\delta$, the circles $B\left(\lambda_{k(p)}, \delta \lambda_{k(p)}\right), p=1,2, \ldots$, are mutually disjoint. This is why the sets $\Lambda_{p}, p=1,2, \ldots$, are mutually disjoint, too. We shall assume that the elements $\mu_{m}$ of sequence $\tilde{\Lambda}$ are indexed in the ascending order. By $m(p)$, $p=1,2, \ldots$, we denote the number for which $\mu_{m(p)}=\lambda_{k(p)}$. The inequalities

$$
\ln \left|q_{\tilde{\Lambda}}^{m(p)}\left(\mu_{m(p)}, \delta\right)\right| \leqslant-6 \tau \delta \mu_{m(p)}, \quad p=1,2, \ldots
$$


hold true. Indeed, the function $q_{\tilde{\Lambda}}^{m(p)}$ comprises the multipliers constructed by the points of set $\Lambda_{p}$. If it coincides with the set of points in $\Lambda$ lying in circle $B\left(\lambda_{k(p)}, \delta \lambda_{k(p)}\right)$, then $q_{\tilde{\Lambda}}^{m(p)}\left(\mu_{m(p)}, \delta\right)=$ $q_{\Lambda}^{k(p)}\left(\lambda_{k(p)}, \delta\right)$ and (13) are implied by (11). Otherwise, taking into consideration (12) and inequality $\delta<1 / 4$, as in Lemma 1 , we get

$$
\ln \left|q_{\tilde{\Lambda}}^{m(p)}\left(\mu_{m(p)}, \delta\right)\right| \leqslant-(l(p)-1) \ln (3(1-\delta)) \leqslant(-(l(p)-1)) / 2 \leqslant-6 \tau \delta \mu_{m(p)} .
$$

Let us show now that the upper density of sequence $\tilde{\Lambda}$ is finite. According to (12), the inequalities

$$
\frac{n\left((1+\delta) \mu_{m(p)}, \tilde{\Lambda}\right)-n\left((1-\delta) \mu_{m(p)}, \tilde{\Lambda}\right)}{\mu_{m(p)}} \leqslant 12 \tau \delta+\frac{2}{\mu_{m(p)}}, \quad p=1,2, \ldots
$$

hold true. Let $r>0$ and $p(r)$ be the maximal among the numbers $p=1,2, \ldots$, for which the intervals $(0, r)$ and $\left((1-\delta) \mu_{m(p)},(1+\delta) \mu_{m(p)}\right)$ intersect. Then the inequality $r>(1-\delta) \mu_{m(p(r))}$ is valid. Since all the points $\mu_{m}$ lie in the union $\cup_{p=1}^{\infty}\left((1-\delta) \mu_{m(p)},(1+\delta) \mu_{m(p)}\right)$ and $\mu_{m(p)}=\lambda_{k(p)}$, $p=1,2, \ldots$, due to (14) and (11) we get

$$
\begin{aligned}
\frac{n(r, \tilde{\Lambda})}{r} & \leqslant \sum_{p=1}^{p(r)} \frac{n\left((1+\delta) \mu_{m(p)}, \tilde{\Lambda}\right)-n\left((1-\delta) \mu_{m(p)}, \tilde{\Lambda}\right)}{r} \leqslant \sum_{p=1}^{p(r)} \frac{12 \tau \delta \mu_{m(p)}+2}{r} \leqslant \\
& \leqslant \sum_{p=1}^{p(r)} \frac{12 \tau \delta \mu_{m(p)}+2}{(1-\delta) \mu_{m(p(r))}} \leqslant \frac{p(r)}{(1-\delta) 2^{p(r)-2} \mu_{m(1)}}+\sum_{p=1}^{p(r)} \frac{12 \tau \delta}{(1-\delta) 2^{p(r)-p}} .
\end{aligned}
$$

It follows that quantity $\bar{\tau}(\tilde{\Lambda})$ is finite.

Let us find the upper estimates for the absolute values of functions $g_{p}$. Since $a_{p} \geq 1$, we have

$$
\begin{gathered}
\left|a_{p} q_{\tilde{\Lambda}}^{m(p)}(\lambda, \delta)\right| \geq\left|q_{\tilde{\Lambda}}^{m(p)}(\lambda, \delta)\right|=\left|\prod_{\mu_{m} \in B\left(\mu_{m(p)}, \delta \mu_{m(p)}\right), m \neq m(p)} \frac{\left(\lambda-\mu_{m}\right)}{3 \delta \mu_{m}}\right| \geq \\
\geq \prod_{\mu_{m} \in B\left(\mu_{m(p)}, \delta \mu_{m(p)}\right), m \neq m(p)} \frac{4 \delta \mu_{m(p)}}{3 \delta \mu_{m}} \geq 1, \quad \lambda \in S\left(\mu_{m(p)}, 5 \delta \mu_{m(p)}\right) .
\end{gathered}
$$

Therefore, the inequalities

$$
\begin{gathered}
\quad\left|g_{p}(z)\right|=\left|\frac{1}{2 \pi i} \int_{\lambda \in S\left(\mu_{m(p)}, 5 \delta \mu_{m(p)}\right)} \frac{\exp (\lambda z) d \lambda}{a_{p}\left(\lambda-\mu_{m(p)}\right) q_{\tilde{\Lambda}}^{m(p)}\left(\mu_{m(p)}, \delta\right)}\right| \leqslant \\
\leqslant \sup _{\lambda \in S\left(\mu_{m(p)}, 5 \delta \mu_{m(p)}\right)}|\exp (\lambda z)| \leqslant \exp \left(\operatorname{Re}\left(\mu_{m(p)} z\right)+5 \delta \mu_{m(p)}|z|\right), \quad z \in \mathbb{C} .
\end{gathered}
$$

hold true.

Let us show that series (9) converge uniformly on compact subsets of the domain $D=\{z$ : $\operatorname{Re} z<\tau \delta\} \cap B(0, \tau)$. Let $z \in D$ and $|z| \leqslant \tau-\beta$. The latter estimates, the definition of coefficients $c_{p}$ and (11) yield

$$
|g(z)| \leqslant \sum_{p=1}^{\infty} \exp \left(\operatorname{Re}\left(\mu_{m(p)} z\right)+5 \delta \mu_{m(p)}|z|-6 \tau \delta \mu_{m(p)}\right) \leqslant \sum_{p=1}^{\infty} \exp \left(-5 \beta \delta \mu_{m(p)}\right)<\infty .
$$

The terms of series (9) are entire functions. Therefore, $g(z)$ is function analytic in domain $D$. We note that it is true for each choice of numbers $a_{p} \geq 1, p=1,2, \ldots$ Let us show now that under a suitable choice of these numbers, function $g(z)$ can be expanded in the Dirichlet series 
whose line of convergence coincides with the imaginary axis. Employing the theory of residues, for each $p=1,2, \ldots$ we obtain

$$
g_{p}(z)=a_{p}^{-1}\left(b_{m(p)} \exp \left(\mu_{m(p)} z\right)+\sum_{\mu_{m} \in \Lambda_{p}, m \neq m(p)} b_{m} \exp \left(\mu_{m} z\right)\right),
$$

where $b_{m(p)}=\left(q_{\tilde{\Lambda}}^{m(p)}\left(\mu_{m(p)}, \delta\right)\right)^{-1}$. Let $p=1,2, \ldots$ For each number $m$ such that $\mu_{m} \in \Lambda_{p}$, we let $d_{m}=c_{p} b_{m} a_{p}{ }^{-1}$. By (13) and the definition of $c_{p}$, the inequality

$$
\max _{m: \mu_{m} \in \Lambda_{p}} \frac{\ln \left|c_{p} b_{m}\right|}{\mu_{m(p)}} \geq \frac{-6 \tau \delta \mu_{m(p)}-\ln \left|q_{\tilde{\Lambda}}^{m(p)}\left(\mu_{m(p)}, \delta\right)\right|}{\mu_{m(p)}} \geq 0
$$

holds true. This is why there exists a number $a_{p} \geq 1$ so that

$$
\max _{m: \mu_{m} \in \Lambda_{p}} \frac{\ln \left|d_{m}\right|}{\mu_{m(p)}}=\max _{m: \mu_{m} \in \Lambda_{p}} \frac{\ln \left|c_{p} b_{m}\right|-\ln a_{p}}{\mu_{m(p)}}=0 .
$$

Consider the Dirichlet series

$$
\sum_{m=1}^{\infty} d_{m} \exp \left(\mu_{m} z\right)
$$

Since the upper density $\bar{\tau}(\tilde{\Lambda})$ is finite, it is obvious that the quantity $\lim _{m \rightarrow \infty} \ln m / \mu_{m}$ vanishes. This is why (see, for instance, [1, Ch. II, Sect. 1, Th. 2.1.2], [11]) Cauchy-Hadamard formula

$$
\gamma=\varlimsup_{m \rightarrow \infty} \frac{\ln \left|d_{m}\right|}{\mu_{m}}
$$

holds true for series (16), where $\gamma$ is the convergence abscissa for this series. At that, in the half-plane $\{z \in \mathbb{C}: \operatorname{Re} z<\gamma\}$, series (16) converges absolutely (see, for instance, [1, Ch. II, Sect. 1, Cor. of Th. 2.1.1], [11]). By (15),

$$
\gamma=\varlimsup_{m \rightarrow \infty} \frac{\ln \left|d_{m}\right|}{\mu_{m}}=\varlimsup_{p \rightarrow \infty} \max _{m: \mu_{m} \in \Lambda_{p}} \frac{\ln \left|d_{m}\right|}{\mu_{m}}=0 .
$$

Thus, the imaginary axis is the convergence line for series (16). Since it converges absolutely in the left half-plane, the sums of series (9) and (16) coincide in the intersection $\{z \in \mathbb{C}: \operatorname{Re} z<$ $0\} \cap D$. It means that function $g \in W(\Lambda, G)$ can be represented series (1) with convergence abscissa $\gamma=0$. The proof is complete.

Let $\Lambda=\left\{\lambda_{k}\right\}_{k=1}^{\infty}, \tilde{\Lambda}=\left\{\mu_{n}\right\}_{n=1}^{\infty}$. We say that $\Lambda$ is a part of $\tilde{\Lambda}(\Lambda \subset \tilde{\Lambda})$ or $\tilde{\Lambda}$ is the completion of $\Lambda$ if there exists a subsequence $\left\{\mu_{n(k)}\right\}$ coinciding with $\left\{\lambda_{k}\right\}$. The well-known Pólya theorem (see, for instance, [9, Ch. VI, Sect. E3]) states that each sequence with a finite maximal density is a part of some measurable sequence with the same density. Since this result is important for further studies and understanding the whole picture, we provide its proof. The method employed for constructing the completion, in our opinion, is simpler than that in book [9].

Lemma 5 (Pólya theorem). Suppose the maximal density of a sequence $\Lambda=\left\{\lambda_{k}\right\}_{k=1}^{\infty}$ is finite. Then there exists its measurable completion $\tilde{\Lambda}$ such that $\tau(\tilde{\Lambda})=\tau_{0}(\Lambda)$.

Proof. We shall seek sequence $\tilde{\Lambda}$ as the union $\cup_{m=1}^{\infty} \Lambda_{m}$, where $\Lambda_{m}=\left\{\mu_{n}, n(m) \leqslant n<n(m+1)\right\}$, $m=1,2, \ldots$ and $n(1)=1$. We construct sets $\Lambda_{m}$ by induction. Let $\alpha=1 / \tau_{0}(\Lambda)$ and $m=1$. If the semi-interval $(0, \alpha]$ contains the points in $\Lambda$, we let $\mu_{n}=\lambda_{n}, 1 \leqslant n<n(2)$, where $n(2)$ is the minimal of numbers $k$ satisfying the inequality $\lambda_{k}>\alpha$. Otherwise we let $\mu_{1}=\alpha$ and $n(2)=2$. Suppose we have constructed sets $\Lambda_{m}$ for $m<p$. Let us define $\Lambda_{p}$. By $s_{p}$ we indicate the number of points in sequence $\Lambda$ lying in the semi-interval $(\alpha(p-1), \alpha p]$ (it can happen that $\left.s_{p}=0\right)$. If the total amount of points in the sets $\Lambda_{1}, \ldots, \Lambda_{p-1}$ does not exceed $p-1$ and $s_{p}=0$, we let $n(p+1)=n(p)+1$ and $\Lambda_{p}=\left\{\mu_{n(p)}\right\}$, where $\mu_{n(p)}=\alpha p$. Otherwise, as $\Lambda_{p}$, we take the 
set consisting of all points $\Lambda$ lying in $\left(\alpha(p-1), \alpha p\right.$ ] (if $s_{p}=0$, set $\Lambda_{p}$ is empty). At that, we let $n(p+1)=n(p)+s_{p}$.

Let us show that $\tilde{\Lambda}$ is the desired sequence. Let $\lambda_{k}$ be an arbitrary point in $\Lambda$ and a number $m$ is so that the semi-interval $(\alpha(m-1), \alpha m]$ contains $\lambda_{k}$. Then $s_{m} \neq 0$ and by construction, set $\Lambda_{m}$ together with sequence $\tilde{\Lambda}$ comprise $\lambda_{k}$. Therefore, $\Lambda \subset \tilde{\Lambda}$. Employing induction, let us prove the inequalities

$$
n(\alpha m, \tilde{\Lambda}) \geq m, \quad m=1,2, \ldots
$$

By construction, each such semi-interval $(\alpha(m-1), \alpha m]$ intersects with $\tilde{\Lambda}$ by the set $\Lambda_{m}$. This is why $n(\alpha m, \tilde{\Lambda})$ coincides with the total number of points in the sets $\Lambda_{1}, \ldots, \Lambda_{m}$. Let $m=1$. Then $n(\alpha, \tilde{\Lambda})=1$ if $s_{1}=0$, and $n(\alpha, \tilde{\Lambda})=s_{1} \geq 1$ otherwise. Suppose (17) is proven for all $m<p$. If $n(\alpha(p-1), \tilde{\Lambda})>p-1$, then $n(\alpha p, \tilde{\Lambda}) \geq n(\alpha(p-1), \tilde{\Lambda}) \geq p$. Let $n(\alpha(p-1), \tilde{\Lambda}) \leqslant p-1$. Then due to $(17)$ we obtain $n(\alpha(p-1), \tilde{\Lambda})=p-1$. In this case, by construction, $n(\alpha p, \tilde{\Lambda})=n(\alpha(p-1), \tilde{\Lambda})+1=p$, if $s_{p}=0$, and $n(\alpha p, \tilde{\Lambda})=n(\alpha(p-1), \tilde{\Lambda})+s_{p} \geq p$ otherwise. Hence, inequality (17) is valid for all $m$. Taking it into account, we obtain

$$
\underline{\tau}(\tilde{\Lambda})=\lim _{r \rightarrow \infty} \frac{n(r, \tilde{\Lambda})}{r} \geq \lim _{p \rightarrow \infty} \frac{n(\alpha p(r), \tilde{\Lambda})}{\alpha p(r)+\beta(r)}=\lim _{p \rightarrow \infty} \frac{n(\alpha p, \tilde{\Lambda})}{\alpha p} \geq \frac{1}{\alpha}=\tau_{0}(\Lambda),
$$

where $\beta(r) \in(0, \alpha]$ as $r \geq 1$. It remains to prove inequality $\bar{\tau}(\tilde{\Lambda}) \leqslant \tau_{0}(\Lambda)$. Suppose $\bar{\tau}(\tilde{\Lambda}) \geq$ $\tau_{0}(\Lambda)+3 \varepsilon$ for some $\varepsilon>0$. Then due to $(2), \tau_{0}(\tilde{\Lambda}) \geq \tau_{0}(\Lambda)+3 \varepsilon$. It was observed above that in the definition of the maximal density one can take the limit instead of the upper limit w.r.t. $\delta$. This is why there exists $\delta_{0}>0$ such that

$$
\varlimsup_{r \rightarrow \infty} \frac{n(r, \tilde{\Lambda})-n((1-\delta) r, \tilde{\Lambda})}{\delta r} \geq \tau_{0}(\Lambda)+2 \varepsilon, \quad \delta \in\left(0, \delta_{0}\right) .
$$

Lessening $\delta_{0}>0$ if necessary, we can assume that the inequality

$$
\varlimsup_{r \rightarrow \infty} \frac{n(r, \Lambda)-n((1-\delta) r, \Lambda)}{\delta r} \leqslant \tau_{0}(\Lambda)+\varepsilon, \quad \delta \in\left(0, \delta_{0}\right)
$$

holds true as well. We fix $\delta \in\left(0, \delta_{0}\right)$. If for some $r>0$, the semi-interval $((1-\delta) r, r]$ contains no points $\tilde{\Lambda}$ different to $\Lambda$, then $n(r, \tilde{\Lambda})-n((1-\delta) r, \tilde{\Lambda})=n(r, \Lambda)-n((1-\delta) r, \Lambda)$. We note that by construction, all the points $\tilde{\Lambda}$ not belonging $\Lambda$ read as $\mu_{n(p)}=\alpha p$, where $p$ ranges in a some subsequence $P$ of natural numbers. Thus, in view of (19) and (20), for each sufficiently large $r>0$, there exists a maximal number $p(r) \in P$ such that $\alpha p(r) \in((1-\delta) r, r]$. Due to the maximal property $p(r)$, each non-empty semi-interval $(\alpha p(r), r]$ contains no points $\tilde{\Lambda}$ different to points $\Lambda$. This is why

$$
n(r, \tilde{\Lambda})-n(\alpha p(r), \tilde{\Lambda})=n(r, \Lambda)-n(\alpha p(r), \Lambda) .
$$

While proving inequality (18), we have shown that $n(\alpha p(r), \tilde{\Lambda})=p(r)$. Therefore,

$$
\varlimsup_{r \rightarrow \infty} \frac{n(r, \tilde{\Lambda})-n((1-\delta) r, \tilde{\Lambda})}{\delta r} \leqslant \varlimsup_{j \rightarrow \infty} \frac{n\left(r_{j}, \Lambda\right)-n\left(\alpha p\left(r_{j}\right), \Lambda\right)}{\delta r_{j}}+\varlimsup_{r \rightarrow \infty} \frac{p\left(r_{j}\right)-n\left((1-\delta) r_{j}, \tilde{\Lambda}\right)}{\delta r_{j}},
$$

where $r_{j} \rightarrow \infty$ implements the left upper limit in this inequality. Since $\alpha p(r) \in((1-\delta) r, r]$, passing to a subsequence, we can assume that $\alpha p\left(r_{j}\right) / r_{j}$ converges to some $\alpha \gamma \in[1-\delta, 1]$. Then in view of $(20)$ we obtain

$$
\begin{gathered}
\varlimsup_{j \rightarrow \infty} \frac{n\left(r_{j}, \Lambda\right)-n\left(\alpha p\left(r_{j}\right), \Lambda\right)}{\delta r_{j}} \leqslant \varlimsup_{j \rightarrow \infty} \frac{n\left(r_{j}, \Lambda\right)-n\left((\alpha \gamma-\tilde{\delta}) r_{j}, \Lambda\right)}{\delta r_{j}}= \\
=(1-\alpha \gamma+\tilde{\delta}) \varlimsup_{j \rightarrow \infty} \frac{n\left(r_{j}, \Lambda\right)-n\left((\alpha \gamma-\tilde{\delta}) r_{j}, \Lambda\right)}{(1-\alpha \gamma+\tilde{\delta}) \delta r_{j}} \leqslant \frac{(1-\alpha \gamma+\tilde{\delta})}{\delta}\left(\tau_{0}(\Lambda)+\varepsilon\right),
\end{gathered}
$$


where $\tilde{\delta}>0$ is such that $\delta+\tilde{\delta} \in\left(0, \delta_{0}\right)$. Let $m_{j}$ be a maximal natural number such that $\alpha m_{j} \leqslant(1-\delta) r_{j}$. By (17) we have

$$
\varlimsup_{j \rightarrow \infty} \frac{p\left(r_{j}\right)-n\left((1-\delta) r_{j}, \tilde{\Lambda}\right)}{\delta r_{j}} \leqslant \varlimsup_{j \rightarrow \infty} \frac{p\left(r_{j}\right)-n\left(\alpha m_{j}, \tilde{\Lambda}\right)}{\delta r_{j}}=\frac{\gamma}{\delta}-\frac{(1-\delta)}{\delta \alpha} .
$$

Thus, we obtain

$$
\begin{gathered}
\varlimsup_{r \rightarrow \infty} \frac{n(r, \tilde{\Lambda})-n((1-\delta) r, \tilde{\Lambda})}{\delta r} \leqslant \frac{(1-\alpha \gamma+\tilde{\delta})}{\delta}\left(\tau_{0}(\Lambda)+\varepsilon\right)+\frac{\gamma}{\delta}-\frac{(1-\delta)}{\delta \alpha}= \\
=\frac{\tilde{\delta}\left(\tau_{0}(\Lambda)+\varepsilon\right)+(1-\alpha \gamma) \varepsilon}{\delta}+\tau_{0}(\Lambda) \leqslant \frac{\tilde{\delta}\left(\tau_{0}(\Lambda)+\varepsilon\right)}{\delta}+\varepsilon+\tau_{0}(\Lambda) .
\end{gathered}
$$

Since $\tilde{\delta}$ can be regarded as infinitesimal, the latter inequality contradicts (19). Thus, our assumption is wrong, i.e., $\bar{\tau}(\tilde{\Lambda}) \leqslant \tau_{0}(\Lambda)$. Together with (18) it completes the proof.

Remark. The proof of the lemma remains true if instead of strictly increasing sequence $\Lambda$, one takes a non-decaying multiple sequence.

Lemma 6. Let $\Lambda=\left\{\lambda_{k}\right\}_{k=1}^{\infty}$ be an unbounded strictly increasing sequence of positive numbers with a finite maximal density $\tau_{0}(\Lambda)$. Then there exists an entire function $f$ of exponential type and regular growth vanishing at the points $\lambda_{k}, k \geq 1$, whose adjoint diagram is the segment of imaginary axis $\left[-i \pi \tau_{0}(\Lambda), i \pi \tau_{0}(\Lambda)\right]$. At that, for all $\lambda$ not lying on the real axis, the identity

$$
\lim _{t \rightarrow+\infty} \frac{\ln |f(t \lambda)|}{t}=\pi \tau_{0}(\Lambda)|\operatorname{Im} \lambda|
$$

holds true and the convergence is uniform the angle $\alpha<\varphi<\pi-\alpha, \alpha \in(0, \pi)$ for all $\lambda=$ $\exp (i \varphi)$.

Proof. By Lemma 5, there exists the completion $\tilde{\Lambda}=\left\{\mu_{n}\right\}_{n=1}^{\infty}$ of sequence $\Lambda$ such that $\tau(\tilde{\Lambda})=$ $\tau_{0}(\Lambda)$. Consider the function

$$
f(\lambda)=\prod_{n=1}^{\infty}\left(1-\frac{\lambda^{2}}{\mu_{n}^{2}}\right) .
$$

It vanishes at the points $\lambda_{k}, k \geq 1$. Since $\tilde{\Lambda}$ is measurable, it is easy to check that the zero set $f(\lambda)$ is well-distributed. This is why $f$ has a regular growth. By Theorem 5.9 in book $[7$, Ch. I, Sect. 5], the required identity holds true that implies immediately that the segment $\left[-i \pi \tau_{0}(\Lambda), i \pi \tau_{0}(\Lambda)\right]$ is the adjoint diagram of function $f$. The proof is complete.

Let $R \subset \mathbb{C}$ and $\delta>0$. By $R^{\delta}$ we denote the union of the circles $B(z, \delta|z|)$, where $z$ ranges in set $R$.

Lemma 7. Let $D$ be a convex domain in $\mathbb{C}, \Lambda=\left\{\lambda_{k}\right\}_{k=1}^{\infty}$ be an unbounded strictly increasing sequence of positive numbers with a finite maximal density $\tau_{0}(\Lambda)$. Suppose space $W(\Lambda, D)$ is nontrivial, $H_{D}(1)<+\infty$, and the intersection of the support line $\left\{z \in \mathbb{C}: \operatorname{Re} z=H_{D}(1)\right\}$ with the boundary of domain $D$ containing the segment of length $2 \pi \tau_{0}(\Lambda)$. Then for each compact set $L \subset D$ and each $\delta>0$, there exists function $f \in P_{D}$ vanishing at the points $\lambda_{k}, k \geq 1$, and such that for some $T(\delta)>0$, the ray $(T(\delta),+\infty)$ lies on the set $R^{\delta}$, where $R=\left\{z: \ln |f(z)| \geq H_{L}(z)\right\}$.

Proof. By assumption, subspace $W(\Lambda, D)$ is nontrivial. This is why, as it was mentioned above, there exists an entire function $f_{1} \in P_{D}$ vanishing at the points $\lambda_{k}, k \geq 1$. Let $K_{1}$ be the adjoint diagram of function $f_{1}$. Since $K_{1}$ is a compact set in domain $D$, the inequality

$$
H_{K_{1}}(z)<H_{D}(z), \quad z \neq 0
$$

holds true. 
According to Lemma 6 , there exists a function $\tilde{f}_{2}$ of exponential type vanishing at the points $\lambda_{k}, k \geq 1$ whose adjoint diagram is $K=\left[-\pi \tau_{0}(\Lambda), \pi \tau_{0}(\Lambda)\right]$. Moreover, for all $z$ not lying on the real axis, we have

$$
\lim _{t \rightarrow+\infty} \frac{\ln \left|\tilde{f}_{2}(t z)\right|}{t}=\pi \tau_{0}(\Lambda)|\operatorname{Im} z|
$$

and the convergence is uniform in the angle $\alpha<\varphi<\pi-\alpha, \alpha \in(0, \pi)$ for all $z=\exp (i \varphi)$. The hypothesis implies that for some $w_{0} \in \mathbb{C}$, the segment $K_{2}=K+w_{0}$ lies on the boundary of domain $D$. Therefore, the inequality

$$
H_{K_{2}}(z) \leqslant H_{D}(z), \quad z \in \mathbb{C},
$$

holds true. Compact set $K_{2}$ is the adjoint diagram of $f_{2}(z)=\tilde{f}_{2}(z) \exp \left(w_{0} z\right)$, which by Lemma 6 is an entire function of exponential type and regular growth. Moreover, it vanishes at the points $\lambda_{k}, k \geq 1$. Consider the function

$$
f_{\Lambda}(z)=\prod_{k=1}^{\infty}\left(1-\frac{z}{\lambda_{k}}\right) \exp \left(\frac{z}{\lambda_{k}}\right) .
$$

It is entire, has the first order of growth and possibly infinite type (see, for instance, [1, Ch. I, Sect. 1, Thms. 1.1.3 and 1.1.5). Since $f_{1}$ and $f_{2}$ are divisible by $f_{\Lambda}$, the functions $\ln \left|f_{1}\right|-\ln \left|f_{\Lambda}\right|$ and $\ln \left|f_{2}\right|-\ln \left|f_{\Lambda}\right|$ are subharmonic in the plane and has the first order of growth (see [5, Ch. I, Sect. 9, Cor. of Thm. 12]). Then by Theorem 5 in work [12], for each $\tau \in(0,1)$, there exist an entire function $\varphi_{\tau}(z)$, constant $C>0$, and an exceptional set $E \subset \mathbb{C}$ such that

$$
|\ln | \varphi_{\tau}(z)\left|-\psi_{\tau}(z)\right| \leqslant C \ln |z|, \quad z \in \mathbb{C} \backslash E,
$$

where $\psi_{\tau}(z)=\tau\left(\ln \left|f_{1}(z)\right|-\ln \left|f_{\Lambda}(z)\right|\right)+(1-\tau)\left(\ln \left|f_{2}(z)\right|-\ln \left|f_{\Lambda}(z)\right|\right)$. At that, $E$ can be covered by the circles $B_{i}=B\left(z_{i}, \gamma_{i}\right), i \geq 1$, such that $\Sigma \gamma_{i}<\infty$. Let $f_{\tau}(z)=\varphi_{\tau}(z) f_{\Lambda}(z)$, $\tau \in(0,1)$. Then $f_{\tau}$ is an entire function.

We fix a compact set $L \subset D$ and a number $\delta>0$ and let us show that as the required function $f$ we can take $f_{\tau}$ for some $\tau \in(0,1)$. First of all, we observe that $f_{\tau}$ vanish at points $\lambda_{k}, k \geq 1$. Then, since $K_{1}$ and $K_{2}$ are adjoint diagrams of functions $f_{1}$ and $f_{2}$, respectively, then in view of the aforementioned Pólya theorem for the indicators in (21), (23), (24) and "smallness" of exceptional set $E$, it is easy to obtain the estimate (see, for instance, [3, Th. 4.3])

$$
h_{f_{\tau}}(z)<H_{D}(z), \quad z \neq 0, \quad \tau \in(0,1) .
$$

It means that the adjoint diagram of $f_{\tau}$ lies in domain $D$, i.e., $f_{\tau} \in P_{D}$ for each $\tau \in(0,1)$. It remains to choose $\tau \in(0,1)$ so that for some $T(\delta)>0$, the ray $(T(\delta),+\infty)$ lies in set $R^{\delta}$, if in the definition of $R$ one takes $f_{\tau}$ as $f$.

By the theorem on the lower estimate for an entire function of finite order and type on the circles (see, for instance, [1, Ch. I, Sect. 1, Thm. 1.1.9]), there exists a number $a>0$ and an unbounded increasing sequence of positive numbers $\left\{r_{p}\right\}_{p=1}^{\infty}$ such that

$$
r_{p+1} \leqslant(1+\delta / 2) r_{p}, \quad \ln \left|f_{1}(z)\right| \geq-a|z|, \quad|z|=r_{p}, p \geq 1 .
$$

Since the segment $K_{2}=K+w_{0}$ lies on the boundary of domain $D$, and segment $K$ contains the origin, point $w_{0}$ lies in the intersection of the boundary of domain $D$ and the support line $\left\{z \in \mathbb{C}: \operatorname{Re} z=H_{D}(1)\right\}$. Therefore, due to the fact that $L$ is a compact set in $D$, there exist $\varepsilon>0$ and $\tilde{\delta} \in(0, \delta / 2)$ so that the inequality

$$
\operatorname{Re}\left(w_{0} z\right)>H_{L}(z)+4 \varepsilon|z|, \quad z \in B(1, \tilde{\delta}),
$$

holds true. Finally, we choose $\tau \in(0,1)$ so that

$$
-\tau \operatorname{Re}\left(w_{0} z\right)>-\varepsilon|z|, \quad-\tau a \geq-\varepsilon .
$$

Since the sum of radii of exceptional circles $B_{i}, i \geq 1$ is finite, there exists an index $p_{0}$ such that for all $p \geq p_{0}$, there exists a point $z_{p} \notin E$ in the arc of the circumference $|z|=r_{p}$ lying 
in the upper half plane and the ring $B\left(r_{p}, \tilde{\delta} r_{p}\right) \backslash B\left(r_{p}, \tilde{\delta} r_{p} / 2\right)$. At that, one can assume that the inequalities $C \ln \left|z_{p}\right|<\varepsilon\left|z_{p}\right|, p \geq p_{0}$ hold true, and by (22), the inequality $\ln \left|\tilde{f}_{2}\left(z_{p}\right)\right|>$ $\pi \tau_{0}(\Lambda)\left|I m z_{p}\right|-\varepsilon\left|z_{p}\right|, p \geq p_{0}$ hold true as well. Then due to (24)-(27), we obtain

$$
\begin{gathered}
\ln \left|f_{\tau}\left(z_{p}\right)\right| \geq \tau \ln \left|f_{1}\left(z_{p}\right)\right|+(1-\tau) \ln \left|f_{2}\left(z_{p}\right)\right|-\varepsilon\left|z_{p}\right| \geq-\tau a\left|z_{p}\right|+ \\
+(1-\tau)\left(\ln \left|\tilde{f}_{2}\left(z_{p}\right)\right|+\operatorname{Re}\left(w_{0} z_{p}\right)\right)-\varepsilon\left|z_{p}\right| \geq-3 \varepsilon\left|z_{p}\right|+(1-\tau) \ln \left|\tilde{f}_{2}\left(z_{p}\right)\right|+\operatorname{Re}\left(w_{0} z_{p}\right) \geq \\
\geq H_{L}\left(z_{p}\right)+4 \varepsilon\left|z_{p}\right|-3 \varepsilon\left|z_{p}\right|-(1-\tau) \varepsilon\left|z_{p}\right|>H_{L}\left(z_{p}\right), \quad p \geq p_{0} .
\end{gathered}
$$

We let $T(\delta)=r_{p_{0}}$ and $z \in(T(\delta),+\infty)$. We choose an index $p \geq p_{0}$ such that $r_{p} \leqslant z<r_{p+1}$. Then by $(25)$ and the choice of $\tilde{\delta}$, we have

$$
\left|z_{p}-z\right| \leqslant\left|z_{p}-r_{p}\right|+\left|r_{p}-z\right|<\tilde{\delta} r_{p}+r_{p+1}-r_{p}<\delta r_{p}=\delta\left|z_{p}\right| .
$$

Thus, in accordance with (28), we get $z \in B\left(z_{p}, \delta\left|z_{p}\right|\right) \subset R^{\delta}$. The proof is complete.

We let

$$
q_{\Lambda}\left(z, \lambda_{k}, \delta\right)=q_{\Lambda}^{k}(z, \delta) \frac{z-\lambda_{k}}{3 \delta \lambda_{k}}, \quad k \geq 1 .
$$

As for functions $q_{\Lambda}^{j}(z, \delta)$, we have the inequality

$$
\left|q_{\Lambda}\left(z, \lambda_{k}, \delta_{1}\right)\right| \geq\left|q_{\Lambda}\left(z, \lambda_{j}, \delta_{2}\right)\right|, \quad z \in B\left(\lambda_{j}, \delta_{2} \lambda_{j}\right),
$$

if $0<\delta_{1} \leqslant \delta_{2}<1 / 3$ and $B\left(\lambda_{k}, \delta_{1} \lambda_{k}\right) \subset B\left(\lambda_{j}, \delta_{2} \lambda_{j}\right)$.

Lemma 8. Let $\Lambda=\left\{\lambda_{k}\right\}_{k=1}^{\infty}$ be an unbounded strictly increasing sequence of positive numbers such that $S_{\Lambda}=0$. Then for $\varepsilon>0$ there exists $\delta \in(0,1 / 3)$ such that for each $\gamma, \theta \in(0,1]$ and some index $k_{0}=k_{0}(\varepsilon, \delta, \gamma, \theta)$, the inequalities

$$
\ln \left|q_{\Lambda}\left(z, \lambda_{k}, \delta\right)\right| \geq-\varepsilon|z|, \quad z \in B\left(\lambda_{k}, \delta\left|\lambda_{k}\right|\right) \backslash \bigcup_{\lambda_{j} \in B\left(\lambda_{k}, \delta \lambda_{k}\right)} B\left(\lambda_{j}, \theta \rho_{j}(\gamma)\right), k \geq k_{0},
$$

hold true, where $\rho_{j}(\gamma)=\min \left\{\gamma / 2,\left(\lambda_{j}-\lambda_{j-1}\right) / 2,\left(\lambda_{j+1}-\lambda_{j}\right) / 2\right\}, j \geq 1$ and $\lambda_{0}=0$.

Proof. Fix $\varepsilon>0$. By the hypothesis, $S_{\Lambda}=0$. Thus, by the definition of $S_{\Lambda}$, there exist $\delta \in(0,1 / 9)$ and an index $k_{1}$ such that

$$
\ln \left|q_{\Lambda}^{j}\left(\lambda_{j}, 3 \delta\right)\right| / \lambda_{j} \geq-\frac{\varepsilon}{6}, \quad j \geq k_{1} .
$$

As in Lemma 1, we obtain

$$
\ln \left|q_{\Lambda}^{j}\left(\lambda_{j}, 3 \delta\right)\right| \leqslant-(m(j, 3 \delta)-1) \ln (3(1-3 \delta)),
$$

where $m(j, 3 \delta)$ is the number of points $\lambda_{k}$ lying in the circle $B\left(\lambda_{j}, 3 \delta \lambda_{j}\right)$. Thus, increasing index $k_{1}$, if necessary, due to (31) and the choice of number $\delta$ we have

$$
m(j, 3 \delta) \leqslant \frac{\varepsilon \lambda_{j}}{6 \ln 2}+1 \leqslant \frac{\varepsilon \lambda_{j}}{3}, \quad j \geq k_{1} .
$$

Let $\gamma, \theta \in(0,1]$ and $z \in S\left(\lambda_{j}, \theta \rho_{j}(\gamma)\right)$. Then by the definition of numbers $\rho_{j}(\gamma)$, for each $l \neq j$, the inequality $\left|z-\lambda_{l}\right| \geq\left|\lambda_{j}-\lambda_{l}\right| / 2$ holds true. Together with (31), (32) it implies

$$
\ln \left|q_{\Lambda}^{j}(z, 3 \delta)\right| \geq \ln \left|q_{\Lambda}^{j}\left(\lambda_{j}, 3 \delta\right)\right|-m(j, 3 \delta) \ln 2 \geq-\varepsilon \lambda_{j} / 2, \quad z \in S\left(\lambda_{j}, \theta \rho_{j}(\gamma)\right), j \geq k_{1} .
$$

If $\rho_{j}(\gamma)=\gamma / 2$, there exists an index $k_{2} \geq k_{1}$ such that

$$
\frac{1}{\lambda_{j}} \ln \left|\frac{z-\lambda_{j}}{9 \delta \lambda_{j}}\right| \geq \frac{1}{\lambda_{j}} \ln \frac{\gamma \theta}{12 \delta \lambda_{j}} \geq-\varepsilon / 4, \quad z \in S\left(\lambda_{j}, \theta \rho_{j}(\gamma)\right), \quad j \geq k_{2} .
$$

Consider now the case $\rho_{j}(\gamma)=\left(\lambda_{j+1}-\lambda_{j}\right) / 2$. Since $\rho_{j}(\gamma) \leqslant 1 / 2$, for some $k_{3} \geq k_{2}$ and all the indices $j \geq k_{3}$ corresponding to this case, the circle $B\left(\lambda_{j+1}, 3 \delta \lambda_{j+1}\right)$ contains point $\lambda_{j}$. This is why one of the terms in the sum determining quantity $\ln \left|q_{\Lambda}^{j+1}(z, 3 \delta)\right|$ is $\ln \left|\left(z-\lambda_{j}\right) / 9 \delta \lambda_{j}\right|$. As it 
was mentioned above, all these terms are non-positive in the circle $B\left(\lambda_{j+1}, 3 \delta \lambda_{j+1}\right)$. Therefore, inequality

$$
\ln \left|\frac{z-\lambda_{j}}{9 \delta \lambda_{j}}\right| \geq \ln \left|q_{\Lambda}^{j+1}(z, 3 \delta)\right|, \quad z \in B\left(\lambda_{j+1}, 3 \delta \lambda_{j+1}\right), \quad j \geq k_{3},
$$

holds true. Hence, by (31),

$$
\ln \frac{\lambda_{j+1}-\lambda_{j}}{9 \delta \lambda_{j}} \geq-\frac{\varepsilon \lambda_{j+1}}{6}, \quad j \geq k_{3} .
$$

It follows that for some $k_{4} \geq k_{3}$

$$
\begin{gathered}
\ln \left|\frac{z-\lambda_{j}}{9 \delta \lambda_{j}}\right|=\ln \frac{\theta \rho_{j}(\gamma)}{9 \delta \lambda_{j}}=\ln \frac{\theta}{2}+\ln \frac{\lambda_{j+1}-\lambda_{j}}{9 \delta \lambda_{j(p)}} \geq \ln \frac{\theta}{2}-\frac{\varepsilon \lambda_{j+1}}{6}= \\
=\ln \frac{\theta}{2}-\frac{\varepsilon\left(\lambda_{j}+2 \rho_{j}(\gamma)\right)}{6} \geq \ln \frac{\theta}{2}-\frac{\varepsilon \lambda_{j}}{6}-\frac{1}{6} \geq-\frac{\varepsilon \lambda_{j}}{4}, \quad z \in S\left(\lambda_{j}, \theta \rho_{j}(\gamma)\right), \quad j \geq k_{4} .
\end{gathered}
$$

The case $\rho_{j}(\gamma)=\left(\lambda_{j}-\lambda_{j-1}\right) / 2$ can be considered in the same way. Thus, we can suppose that in all cases

$$
\ln \left|\frac{z-\lambda_{j}}{9 \delta \lambda_{j}}\right| \geq-\frac{\varepsilon \lambda_{j}}{4}, \quad z \in S\left(\lambda_{j}, \theta \rho_{j}(\gamma)\right), \quad j \geq k_{4}
$$

Together with (33) and the definition of function $q_{\Lambda}\left(z, \lambda_{j}, 3 \delta\right)$ it yields

$$
\ln \left|q_{\Lambda}\left(z, \lambda_{j}, 3 \delta\right)\right| \geq-3 \varepsilon \lambda_{j} / 4, \quad z \in S\left(\lambda_{j}, \theta \rho_{j}(\gamma)\right), \quad j \geq k_{4}
$$

Since $\delta<1 / 3$, for each point $\lambda_{j} \in B\left(\lambda_{k}, \delta\left|\lambda_{k}\right|\right)$, the circle $B\left(\lambda_{j}, 3 \delta\left|\lambda_{j}\right|\right)$ contains the circle $B\left(\lambda_{k}, \delta\left|\lambda_{k}\right|\right)$. We choose an index $k_{0} \geq k_{4}$ such that $j \geq k_{4}$, if $\lambda_{j} \in B\left(\lambda_{k}, \delta\left|\lambda_{k}\right|\right)$ and $k \geq k_{0}$. We can suppose that $S\left(\lambda_{j}, \theta \rho_{j}(\gamma)\right) \subset B\left(\lambda_{j}, 3 \delta\left|\lambda_{j}\right|\right)$ as $j \geq k_{4}$. Then in accordance with (29) and (34) we have

$$
\ln \left|q_{\Lambda}\left(z, \lambda_{k}, \delta\right)\right| \geq-3 \varepsilon \lambda_{j} / 4, \quad z \in S\left(\lambda_{j}, \theta \rho_{j}(\gamma)\right), \quad \lambda_{j} \in B\left(\lambda_{k}, \delta\left|\lambda_{k}\right|\right), k \geq k_{0} .
$$

Therefore, for each $k \geq k_{0}$ and $j$ such that $\lambda_{j} \in B\left(\lambda_{k}, \delta\left|\lambda_{k}\right|\right)$ the inequality

$$
\ln \left|q_{\Lambda}\left(z, \lambda_{k}, \delta\right)\right| \geq-3 \varepsilon(1+\delta) \lambda_{k} / 4 \geq-5 \varepsilon \lambda_{k} / 6, \quad z \in S\left(\lambda_{j}, \theta \rho_{j}(\gamma)\right)
$$

is valid. Moreover, as $\delta<1 / 3$, the inequality

$$
\ln \left|q_{\Lambda}\left(z, \lambda_{k}, \delta\right)\right| \geq 0, \quad z \in S\left(\lambda_{k}, 5 \delta\left|\lambda_{k}\right|\right), \quad k \geq 1,
$$

is valid as well. Then by the maximum principle in the domain $B\left(\lambda_{k}, 5 \delta\left|\lambda_{k}\right|\right) \backslash$ $\cup_{\lambda_{j} \in B\left(\lambda_{k}, \delta \lambda_{k}\right)} B\left(\lambda_{j}, \theta \rho_{j}(\gamma)\right)$, the harmonic function $\ln \left|q_{\Lambda}\left(z, \lambda_{k}, \delta\right)\right|$ satisfies the estimate

$$
\ln \left|q_{\Lambda}\left(z, \lambda_{k}, \delta\right)\right| \geq-5 \varepsilon \lambda_{k} / 6, \quad k \geq k_{0}
$$

Therefore,

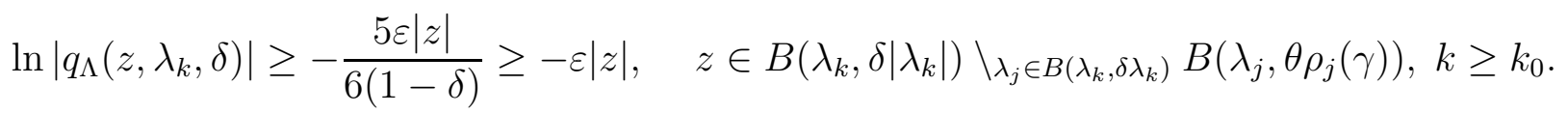

The proof is complete.

Lemma 9. Let $\Lambda=\left\{\lambda_{k}\right\}_{k=1}^{\infty}$ be an unbounded strictly increasing sequence of positive numbers such that $S_{\Lambda}=0$. Then for each $\tilde{\varepsilon}>0$, there exists its completion $\Lambda_{\tilde{\varepsilon}}=\left\{\mu_{n}\right\}_{n=1}^{\infty} \subset(0,+\infty)$ $\left(\Lambda_{\tilde{\varepsilon}}\right.$ is strictly increasing) and a number $\gamma_{0} \in(0,1)$ such that the inequalities

$$
|\ln | f(\lambda)\left|-\frac{\pi|\operatorname{Im} \lambda|}{\gamma_{0}}\right| \leqslant \tilde{\varepsilon}|\lambda|, \quad \lambda \in \mathbb{C} \backslash\left(E_{1} \cup E_{2} \cup B(0, T)\right)
$$

hold true, where $f$ is an entire function of exponential type,

$$
f(\lambda)=\prod_{n=1}^{\infty}\left(1-\frac{\lambda^{2}}{\mu_{n}^{2}}\right) \quad, E_{1}=\bigcup_{k=1}^{\infty} B\left( \pm \lambda_{k}, \theta \rho_{k}\left(\gamma_{0}\right)\right), \quad E_{2}=\bigcup_{n=1}^{\infty} B\left( \pm \tilde{\mu}_{n}\left(\gamma_{0}\right), \theta \gamma_{0} / 2\right),
$$


$\rho_{k}\left(\gamma_{0}\right), k \geq 1$, are defined in Lemma $8, \tilde{\mu}_{n}\left(\gamma_{0}\right)=\gamma_{0} n-\gamma_{0} / 2, n \geq 1, \theta \in(0,1)$ and $T>0$ depend on $\theta, \tilde{\varepsilon}$.

Proof. Since $S_{\Lambda}=0$, by Lemma 2, upper density $\bar{\tau}(\Lambda)$ is finite. Let $\gamma>0$. First we construct the completion $\Lambda(\gamma)=\left\{\mu_{n}(\gamma)\right\}_{n=1}^{\infty}$ of sequence $\Lambda$. We shall seek it as the union $\cup_{m=1}^{\infty} \Lambda_{m}(\gamma)$, where $\Lambda_{m}(\gamma)=\left\{\mu_{n}(\gamma), n(m) \leqslant n<n(m+1)\right\}, m=1,2, \ldots$, and $n(1)=1$. We construct sets $\Lambda_{m}(\gamma)$ by induction. Let $m=1$. If the semi-interval $(0, \gamma]$ contains points in $\Lambda$, we let $\mu_{n}(\gamma)=\lambda_{n}, 1 \leqslant n<n(2)$, where $n(2)$ is the minimal of indices $k$ satisfying the inequality $\lambda_{k}>\gamma$. Otherwise we let $\mu_{1}(\gamma)=\gamma / 2$ and $n(2)=2$. Suppose we have already constructed sets $\Lambda_{m}(\gamma)$ for all $m<p$. Let us define $\Lambda_{p}(\gamma)$. We indicate $s_{p}$ the number of the points of sequence $\Lambda$ lying in the semi-interval $\left(\gamma(p-1), \gamma p\right.$ ] (it can happen that $\left.s_{p}=0\right)$. If the total number of points in the sets $\Lambda_{1}(\gamma), \ldots, \Lambda_{p-1}(\gamma)$ does not exceed $p-1$ and $s_{p}=0$, we let $n(p+1)=n(p)+1$ and $\Lambda_{p}(\gamma)=\left\{\mu_{n(p)}(\gamma)\right\}$, where $\mu_{n(p)}(\gamma)=\gamma p-\gamma / 2$. Otherwise as $\Lambda_{p}(\gamma)$, we take the set consisting of all points $\Lambda$ lying in $(\gamma(p-1), \gamma p]$ (if $s_{p}=0$, then $\Lambda_{p}(\gamma)$ is empty). Here we let $n(p+1)=n(p)+s_{p}$.

Let $\lambda_{k}$ be an arbitrary point in $\Lambda$, and the index $m$ is so that the semi-interval $(\gamma(m-1), \gamma m]$ contains $\lambda_{k}$. Then $s_{m} \neq 0$ and by construction, set $\Lambda_{m}(\gamma)$ together with sequence $\Lambda(\gamma)$ contain $\lambda_{k}$. Therefore, $\Lambda \subset \Lambda(\gamma)$. Employing the induction, let prove inequalities

$$
n(\gamma m, \Lambda(\gamma)) \geq m, \quad m=1,2, \ldots
$$

By construction, each semi-interval $(\gamma(m-1), \gamma m]$ intersects $\Lambda(\gamma)$ by set $\Lambda_{m}(\gamma)$. This is why $n(\gamma m, \Lambda(\gamma))$ coincides with the total amount of the points in sets $\Lambda_{1}(\gamma), \ldots, \Lambda_{m}(\gamma)$. Let $m=1$. Then $n(\gamma, \Lambda(\gamma))=1$, if $s_{1}=0$, and $n(\gamma, \Lambda(\gamma))=s_{1} \geq 1$ otherwise. Suppose (36) is proven for all $m<p$. If $n(\gamma(p-1), \Lambda(\gamma))>p-1$, then $n(\gamma p, \Lambda(\gamma)) \geq n(\gamma(p-1), \Lambda(\gamma)) \geq p$. Let $n(\gamma(p-1), \Lambda(\gamma)) \leqslant p-1$. Then by induction assumption we get $n(\gamma(p-1), \Lambda(\gamma))=p-1$. In this case by construction, $n(\gamma p, \Lambda(\gamma))=n\left(\gamma(p-1), \Lambda(\gamma)+1=p\right.$, if $s_{p}=0$, and $n(\gamma p, \Lambda(\gamma))=$ $n(\gamma(p-1), \Lambda(\gamma))+s_{p} \geq p$ otherwise. Thus, inequality (36) is valid for all $m$.

By construction, each of groups $\Lambda_{m}(\gamma)$ either comprises only some of points $\lambda_{k}$, or is empty, or consists just of one point $\mu_{n(m)}(\gamma)$ not involved in sequence $\Lambda$. Let us show that as $\gamma<1 / \bar{\tau}(\Lambda)$, there exists infinitely many of the groups of latter kind. Suppose the opposite. Then for some $r_{0}>0$, an infinite interval $\left(r_{0},+\infty\right)$ contains no points of sequence $\Lambda(\gamma)$ different to $\lambda_{k}$. This is why

$$
\varlimsup_{r \rightarrow \infty} \frac{n(r, \Lambda(\gamma))}{r}=\varlimsup_{r \rightarrow \infty} \frac{n(r, \Lambda)-n\left(r_{0}, \Lambda\right)+n\left(r_{0}, \Lambda(\gamma)\right)}{r}=\varlimsup_{r \rightarrow \infty} \frac{n(r, \Lambda)}{r}=\bar{\tau}(\Lambda) .
$$

On the other hand, by (36) we have

$$
\varlimsup_{r \rightarrow \infty} \frac{n(r, \Lambda(\gamma))}{r} \geq \varlimsup_{m \rightarrow \infty} \frac{n(\gamma m, \Lambda(\gamma))}{\gamma m} \geq \frac{1}{\gamma}>\bar{\tau}(\Lambda) .
$$

We obtain the contradiction. Therefore, there exist infinitely many groups of mentioned kind. Let $I(\gamma)$ be the union of all semi-intervals $(\gamma m, \gamma(m+1)]$ associated with such groups. Then $J(\gamma)=(0,+\infty) \backslash I(\gamma)$ is the union of all bounded semi-intervals $\left(\gamma m_{j}(\gamma), \gamma p_{j}(\gamma)\right], j \geq 1$.

Let $j \geq 1$. According to the definition of $I(\gamma)$, group $\Lambda_{m_{j}(\gamma)}(\gamma)$ consists of the only point $\mu_{n\left(m_{j}(\gamma)\right)}(\gamma)$ not involved in sequence $\Lambda$. Then by construction, we have $n\left(\gamma\left(m_{j}(\gamma)-1\right), \Lambda(\gamma)\right) \leqslant m_{j}(\gamma)-1$ and $s_{m_{j}(\gamma)}=0$. Therefore, $n\left(\gamma m_{j}(\gamma), \Lambda(\gamma)\right) \leqslant m_{j}(\gamma)$. Together with (36) it implies the identity

$$
n\left(\gamma m_{j}(\gamma), \Lambda(\gamma)\right)=m_{j}(\gamma), \quad j \geq 1
$$

Group $\Lambda_{p_{j}(\gamma)+1}(\gamma)$ is analogous to group $\Lambda_{m_{j}(\gamma)}(\gamma)$. Then by construction we have also the identity $n\left(\gamma p_{j}(\gamma), \Lambda(\gamma)\right) \leqslant p_{j}(\gamma)$. By (36) it yields

$$
n\left(\gamma p_{j}(\gamma), \Lambda(\gamma)\right)=p_{j}(\gamma), \quad j \geq 1
$$


Let $\delta^{\prime}>0$. According to the definition of $J(\gamma)$, all the points $\lambda_{k}$ lie in set $J(\gamma)$ and none of the points of sequence $\Lambda(\gamma)$ different to $\lambda_{k}, k \geq 1$, belongs to $J(\gamma)$. Therefore, by (37) and (38), for all $j \geq 1$ we have

$$
p_{j}(\gamma)-m_{j}(\gamma)=n\left(\gamma p_{j}(\gamma), \Lambda(\gamma)\right)-n\left(\gamma m_{j}(\gamma), \Lambda(\gamma)\right)=n\left(\gamma p_{j}(\gamma), \Lambda\right)-n\left(\gamma m_{j}(\gamma), \Lambda\right) .
$$

Since $\Lambda$ has a finite upper density, for some $C>0$ we have the inequality $n(r, \Lambda) \leqslant C r$. This is why it follows from (39) that

$$
p_{j}(\gamma)-m_{j}(\gamma) \leqslant n\left(\gamma p_{j}(\gamma), \Lambda\right) \leqslant C \gamma p_{j}(\gamma) \leqslant \delta^{\prime} p_{j}(\gamma), \quad j \geq 1
$$

for all $\gamma<\min \left\{1 / \bar{\tau}(\Lambda), \delta^{\prime} / C\right\}=\gamma\left(\delta^{\prime}\right)$. It implies easily the estimate

$$
p_{j}(\gamma)-m_{j}(\gamma) \leqslant \delta^{\prime \prime} m_{j}(\gamma), \quad j \geq 1, \quad \gamma<\gamma\left(\delta^{\prime}\right)
$$

where $\delta^{\prime \prime}=\delta^{\prime} /\left(1-\delta^{\prime}\right)$.

We fix $\tilde{\varepsilon}>0$ and $\varepsilon \in(0, \tilde{\varepsilon} / 5)$. Let us show that for some $\gamma>0$, as the required sequence $\Lambda_{\tilde{\varepsilon}}$ we can take $\Lambda(\gamma)$. First of all, according to Lemma 8 , given $\varepsilon>0$, we find $\delta \in(0,1 / 3)$ such that (30) holds true.

We introduce a new sequence $\tilde{\Lambda}(\gamma)=\left\{\tilde{\lambda}_{k}(\gamma)\right\}_{k=1}^{\infty}$ of positive numbers taken in the ascending order. It is the union of the groups $\tilde{\Lambda}_{j}(\gamma), j \geq 1$, where $\tilde{\Lambda}_{j}(\gamma)=\left\{\gamma p_{j}(\gamma)+\gamma / 2, \gamma\left(p_{j}(\gamma)+1\right)+\right.$ $\left.\gamma / 2, \ldots, \gamma m_{j}(\gamma)-\gamma / 2\right\}$. Consider the functions

$$
L(\lambda)=\prod_{k=1}^{\infty}\left(1-\frac{\lambda^{2}}{\lambda_{k}^{2}}\right), \quad L(\lambda, \gamma)=\prod_{k=1}^{\infty}\left(1-\frac{\lambda^{2}}{\left(\tilde{\lambda}_{k}(\gamma)\right)^{2}}\right) .
$$

Sequence $\Lambda$ has a finite upper density. By construction, $\bar{\tau}(\tilde{\Lambda}(\gamma)) \leqslant 1 / \gamma$. This is why (see, for instance, $[1$, Ch. I, Sect. 1, Thm. 1.1.5]) $L(\lambda)$ and $L(\lambda, \gamma)$ are entire functions of exponential type. Let us compare their behavior. In order to do it, we employ the result from work [13]. By (39) and (40), sequence $\tilde{\Lambda}(\gamma)$ is $\delta^{\prime \prime}$-close to $\Lambda$, i.e.,

$$
\left|\lambda_{k}-\tilde{\lambda}_{k}(\gamma)\right| \leqslant \delta^{\prime \prime}\left|\lambda_{k}\right|, \quad k \geq 1,
$$

and thus, it is asymptotically $\delta^{\prime \prime}$-close to sequence $\Lambda$ (in the terminology of work [13]). Since in our case the sums of inverses to zeroes of functions $L(\lambda)$ and $L(\lambda, \gamma)$ lying in the circle $B(0, r)$ equal to zero for each $r>0$, as $\delta^{\prime \prime} \in(0,1 / 2)$, by Theorem in work [13] (where we let $\alpha=1 / 2, \beta=1)$, the inequality

$$
|\ln | L(\lambda)|-\ln | L(\lambda, \gamma)|| \leqslant A \sqrt{\delta^{\prime \prime}}|\lambda|, \quad \lambda \in \mathbb{C} \backslash E\left(\delta^{\prime \prime}\right)
$$

holds true, where $A>0$ is independent of $\delta^{\prime \prime}$. Set $E\left(\delta^{\prime \prime}\right)$ is the union of the circles $B_{i}=B\left(\xi_{i}, \sigma_{i}\right)$, $i \geq 1$ and it has the linear density not exceeding $\sqrt[4]{\delta^{\prime \prime}}$,

$$
\varlimsup_{r \rightarrow \infty} \frac{1}{r} \sum_{\left|\xi_{i}\right| \leqslant r} \sigma_{i} \leqslant \sqrt[4]{\delta^{\prime \prime}}
$$

and it is centered with the union of zero sets for functions $L(\lambda)$ and $L(\lambda, \gamma)$, i.e., each point of these sets lies in at least of circles $B_{i}$, and each of circles $B_{i}$ contains at least one point of this union. We can obviously assume that $E\left(\delta^{\prime \prime}\right)$ is symmetric w.r.t. the origin.

We choose $\delta^{\prime}>0$ such that for $\delta^{\prime \prime}=\delta^{\prime} /\left(1-\delta^{\prime}\right)$, the inequality

$$
A \sqrt{\delta^{\prime \prime}}<\varepsilon, \quad \sqrt[4]{\delta^{\prime \prime}}<\delta / 12
$$

are valid. We fix $\gamma_{0} \in\left(0, \gamma\left(\delta^{\prime}\right)\right), \gamma_{0}<1$, and let us show that sequence $\Lambda_{\tilde{\varepsilon}}=\Lambda\left(\gamma_{0}\right)$ is the required one. Let $\theta \in(0,1)$ and $k_{0}$ be the index for each (30) is satisfied with $\gamma=\gamma_{0}$. According to (43) and (44), we find $r_{0}>0$ such that

$$
\frac{1}{r} \sum_{\left|\xi_{i}\right| \leqslant r} \sigma_{i} \leqslant \delta / 11, \quad r \geq r_{0}
$$


We choose a number $k_{1} \geq k_{0}$ to have $\lambda_{k} \geq r_{0}$ and $\tilde{\lambda}_{k}\left(\gamma_{0}\right) \geq r_{0}$ for each $k \geq k_{1}$, and let $B_{i}$ be an arbitrary circle in set $E\left(\delta^{\prime \prime}\right)$ containing either some point $\lambda_{k}$ or $\tilde{\lambda}_{k}\left(\gamma_{0}\right)$ with an index $k \geq k_{1}$. If $\lambda_{k} \in B_{i}$, according to (45), in the case $\left|\xi_{i}\right| \leqslant \lambda_{k}$ the inequality $\sigma_{i} \leqslant \delta \lambda_{k} / 11$ holds true, while in the case $\left|\xi_{i}\right| \geq \lambda_{k}$ the inequality $\sigma_{i} \leqslant \delta\left|\xi_{i}\right| / 11$ is valid, and then in view of the inclusions $\lambda_{k} \in B_{i}$, $\delta \in(0,1 / 3)$ we obtain $\lambda_{k} \geq\left|\xi_{i}\right|(1-\delta / 11)$ and $\sigma_{i} \leqslant \delta\left|\xi_{i}\right| / 11 \leqslant \delta \lambda_{k} / 11(1-\delta / 11) \leqslant 3 \delta \lambda_{k} / 32$. The situation $\tilde{\lambda}_{k}\left(\gamma_{0}\right) \in B_{i}$ can be treated in the same way. Thus, by (41) and (44), we have

$$
\sigma_{i} \leqslant \max \left\{3 \delta \lambda_{k} / 32,3 \delta \tilde{\lambda}_{k}\left(\gamma_{0}\right) / 32\right\} \leqslant 3 \delta\left(1+\delta^{\prime \prime}\right) \lambda_{k} / 32 \leqslant 13 \delta \lambda_{k} / 128 \leqslant \delta \lambda_{k} / 9 .
$$

It follows that $B_{i}$ lies either in the circle $B\left(\lambda_{k}, 2 \delta \lambda_{k} / 9\right)$ or in the circle $B\left(\tilde{\lambda}_{k}\left(\gamma_{0}\right), 2 \delta \lambda_{k} / 9\right)$. Therefore, in view of (41) and (44), the inclusion $B_{i} \subset B\left(\lambda_{k}, \delta \lambda_{k} / 3\right)$ holds true.

Let $B_{j} \cap B\left(\lambda_{k}, \delta \lambda_{k}\right) \neq \emptyset$. Then by $(45),\left|\xi_{i}\right| \leqslant(1+\delta) \lambda_{k} /(1-\delta / 11) \leqslant 11 \delta \lambda_{k} / 8$. This is why, employing (45) once again, we find that the total sum of all the radii of circles $B_{j}$ intersecting $B\left(\lambda_{k}, \delta \lambda_{k}\right)$ does not exceed $\delta \lambda_{k} / 4$. It means that for $t \in(1 / 2,1)$, the circle $S\left(\lambda_{k}, t \delta \lambda_{k}\right)$ does not intersect set $E\left(\delta^{\prime \prime}\right)$. Then due to (42) and (44) we have the estimate

$$
\ln |L(\lambda)| \geq \ln \left|L\left(\lambda, \gamma_{0}\right)\right|-\varepsilon|\lambda|, \quad \lambda \in S\left(\lambda_{k}, t \delta \lambda_{k}\right), \quad k \geq k_{1} .
$$

We recall that the absolute value of the function $q_{\Lambda}\left(\lambda, \lambda_{k}, \delta\right)$ does not exceeding one in the circle $B\left(\lambda_{k}, \delta\left|\lambda_{k}\right|\right)$. This is why for each $\lambda \in S\left(\lambda_{k}, t \delta \lambda_{k}\right)$ the inequality

$$
\ln \left|L\left(\lambda, \gamma_{0}\right)\right| \leqslant \ln |L(\lambda)|-\ln \left|q_{\Lambda}\left(\lambda, \lambda_{k}, \delta\right)\right|+\varepsilon(1+t \delta) \lambda_{k} \leqslant \ln \left|L(\lambda) / q_{\Lambda}\left(\lambda, \lambda_{k}, \delta\right)\right|+2 \varepsilon \lambda_{k}
$$

holds true, and it can be extended to the circle $B\left(\lambda_{k}, t \delta \lambda_{k}\right)$, since $\ln \left|L(\lambda) / q_{\Lambda}\left(\lambda, \lambda_{k}, \delta\right)\right|$ is a harmonic in $B\left(\lambda_{k}, \delta\left|\lambda_{k}\right|\right)$ function and $\ln \left|L\left(\lambda, \gamma_{0}\right)\right|$ is subharmonic in the same domain. Then by (30) we get

$$
\begin{gathered}
\ln \left|L\left(\lambda, \gamma_{0}\right)\right| \leqslant \ln |L(\lambda)|+2 \varepsilon \lambda_{k}+\varepsilon|\lambda| \leqslant \ln |L(\lambda)|+4 \varepsilon|\lambda|, \\
\lambda \in B\left(\lambda_{k}, t \delta \lambda_{k}\right) \backslash \bigcup_{\lambda_{j} \in B\left(\lambda_{k}, \delta \lambda_{k}\right)} B\left(\lambda_{j}, \theta \rho_{j}\left(\gamma_{0}\right)\right), \quad k \geq k_{1},
\end{gathered}
$$

At that, the union of the circles $B\left( \pm \lambda_{k}, t \delta \lambda_{k}\right), k \geq k_{1}$ cover all the circles $B_{i}$ in set $E\left(\delta^{\prime \prime}\right)$ each of those consists some of the points $\pm \lambda_{k}$ or $\pm \tilde{\lambda}_{k}\left(\gamma_{0}\right)$ with an index $k \geq k_{1}$. It is easy to see that the remaining part of set $E\left(\delta^{\prime \prime}\right)$ (not covered by the mentioned union) lies in the circle $B\left(0, T_{1}\right.$ ) for some $T_{1}>0$. Thus, it follows from (46) that

$$
\ln |L(\lambda)| \geq \ln \left|L\left(\lambda, \gamma_{0}\right)\right|-4 \varepsilon|\lambda|, \quad \lambda \in \mathbb{C} \backslash \bigcup_{k \geq 1} B\left( \pm \lambda_{k}, \theta \rho_{k}\left(\gamma_{0}\right)\right) \cup B\left(0, T_{1}\right) .
$$

We also note that the zeroes of the function $L\left(\lambda, \gamma_{0}\right)$ are separated by the distance at least $\gamma_{0}$. This is why, according to the example discussed before Lemma 1, the quantity $\tilde{S}_{\tilde{\Lambda}\left(\gamma_{0}\right)}$ is finite and thus $S_{\tilde{\Lambda}\left(\gamma_{0}\right)}=0$. Therefore, as to sequence $\Lambda$, we can apply Lemma 8 to sequence $\tilde{\Lambda}\left(\gamma_{0}\right)$ and arguing as above, we can obtain the estimate

$$
\ln \left|L\left(\lambda, \gamma_{0}\right)\right| \geq \ln |L(\lambda)|-4 \varepsilon|\lambda|, \quad \lambda \in \mathbb{C} \backslash \bigcup_{k \geq 1} B\left( \pm \tilde{\lambda}_{k}\left(\gamma_{0}\right), \theta \gamma_{0} / 2\right) \cup B\left(0, T_{2}\right) .
$$

for some $T_{2}>0$.

Consider the functions

$$
f(\lambda)=\prod_{n=1}^{\infty}\left(1-\frac{\lambda^{2}}{\left(\mu_{n}\left(\gamma_{0}\right)\right)^{2}}\right), \quad \tilde{L}\left(\lambda, \gamma_{0}\right)=\prod_{n=1}^{\infty}\left(1-\frac{\lambda^{2}}{\left(\tilde{\mu}_{n}\left(\gamma_{0}\right)\right)^{2}}\right),
$$

where $\tilde{\mu}_{n}\left(\gamma_{0}\right)=\gamma_{0} n-\gamma_{0} / 2, n \geq 1$. It is easy to show that $\Lambda\left(\gamma_{0}\right)$ has a finite upper density not exceeding $\left.\bar{\tau}(\Lambda)+1 / \gamma_{0}\right)$. This is why (see, for instance., [1, Ch. I, Sect. 1, Thm. 1.1.5]) $f(\lambda)$ is an entire function of exponential type. Sequence $\tilde{\Lambda}\left(\gamma_{0}\right)$ has the density $1 / \gamma_{0}$ and is a regular set (see $\left[5\right.$, Ch. II, Sect. 1]). Therefore, $\tilde{L}\left(\lambda, \gamma_{0}\right)$ has a regular growth and its adjoint 
diagram coincides with the segment of imaginary axis $\left[-i \pi / \gamma_{0}, i \pi / \gamma_{0}\right]$ (see $[1$, Ch. I, Sect. 2, Thm. 1.2.9]). Since its zero set $\tilde{L}\left(\lambda, \gamma_{0}\right)$ is regular, the inequality

$$
\ln \left|\tilde{L}\left(\lambda, \gamma_{0}\right)\right|=\frac{\pi|\operatorname{Im} \lambda|}{\gamma_{0}}+o(\lambda), \quad \lambda \in \mathbb{C} \backslash \bigcup_{n \geq 1} B\left( \pm \tilde{\mu}_{n}\left(\gamma_{0}\right), \theta \gamma_{0} / 2\right),
$$

holds true (see [5, Ch. II, Sect. 1, Thm. 5]), $o(\lambda)$ depends on $\theta \in(0,1]$ and $o(\lambda) /|\lambda| \rightarrow 0$ as $|\lambda| \rightarrow \infty$.

By construction,

$$
\ln |f(\lambda)|-\ln \left|\tilde{L}\left(\lambda, \gamma_{0}\right)\right|=\ln |L(\lambda)|-\ln \left|L\left(\lambda, \gamma_{0}\right)\right|, \quad \lambda \in \mathbb{C} .
$$

Moreover, each point $\tilde{\lambda}_{k}\left(\gamma_{0}\right)$ coincides with one of the points $\tilde{\mu}_{n}\left(\gamma_{0}\right)$. This is why in view of the choice of number $\varepsilon>0$ and by (47)-(49) we get (35). The proof is complete.

Lemma 10. Let $D$ be a convex domain in $\mathbb{C}, \Lambda=\left\{\lambda_{k}\right\}_{k=1}^{\infty}$ be an unbounded strictly increasing sequence of positive numbers. Suppose the set of sums of series (1) converging in domain $D$ is closed in space $H(D)$. Then the system $\mathcal{E}=\left\{\exp \left(\lambda_{k} z\right)\right\}_{k=1}^{\infty}$ is incomplete in $H(D)$.

Proof. If the hypothesis of the lemma holds true, by the corollary of Lemma 3 the inequality $\bar{\tau}(\Lambda)<\infty$ holds true. Then, as above, there exists an entire function $f$ of exponential type vanishing at the points $\lambda_{k}, k \geq 1$. Suppose some shift $z_{0}+K$ of the adjoint diagram $K$ for function $f$ lies in domain $D$. Then the function $\exp \left(z_{0} \lambda\right) f(\lambda)$ belongs to space $P_{D}$ and vanishes at the points $\lambda_{k}, k \geq 1$. It means that system $\mathcal{E}$ is incomplete in $H(D)$. It happens, for instance, when $D$ is the plane of a half-plane.

Suppose domain $D$ is neither the plane nor a half-plane and assume system $\mathcal{E}$ is complete in space $H(D)$. Then by the hypothesis, each function in $H(D)$ is represented by series (1) in domain $D$. By Abel theorem for Dirichlet series (see, for instance, [1, Ch. II, Sect. 1, Subsect. 4]), each of such series converges uniformly on compact sets in the half-plane $\left\{z: \operatorname{Re} z<H_{D}(1)\right\}$ (which can coincide with the plane once $H_{D}(1)=+\infty$ ). Therefore, each function in $H(D)$ can be analytically continued into this half-plane that is impossible. Thus, $\mathcal{E}$ is incomplete in $H(D)$. The proof is complete.

\section{MAIN RESUlTS}

Let $D$ be an unbounded convex domain and denote

$$
J(D)=\left\{\lambda \in \mathbb{C}: H_{D}(\lambda)=+\infty\right\} .
$$

If $D=\mathbb{C}$, then $J(D)=\mathbb{C} \backslash\{0\}$. In the case $D$ is a half-plane $\left\{z \in \mathbb{C}: \operatorname{Re}\left(z e^{i \varphi}\right)<a\right\}$, set $J(D)$ is the plane with the cut along the ray $\left\{\lambda=t e^{i \varphi}: t \geq 0\right\}$. If $D$ is the strip $\left\{z \in \mathbb{C}: \operatorname{Re}\left(z e^{i \varphi}\right)<\right.$ $\left.a, \operatorname{Re}\left(z e^{i(\varphi+\pi)}\right)<b\right\}$, then $J(D)=\mathbb{C} \backslash\left\{\lambda=t e^{i \varphi}: t \in \mathbb{R}\right\}$. In all other cases, domain $D$ contains no straight lines. However, $D$ always contains some ray $\left\{z=z_{0}+t e^{i \varphi}, t \geq 0\right\}$. At, set $J(D)$ is an angle strictly less than $2 \pi$ and it involves an open angle of size $\pi$ which the half-plane $\left\{\lambda=t e^{i \psi}:-\varphi-\frac{\pi}{2}<\psi<-\varphi+\frac{\pi}{2}, t>0\right\}$. We shall say that $D$ is narrow, if $D$ is a strip or $J(D)$ coincides with an open half-plane. Otherwise we shall say $D$ is wide.

For a narrow domain $D$, there exists the unique $\psi \in[0, \pi)$, such that $H_{D}\left(e^{i \psi}\right)<+\infty$ and $H_{D}\left(e^{i \psi+\pi}\right)<+\infty$. It corresponds to the boundary rays $\left\{\lambda=t e^{i \psi}: t>0\right\}$ and $\left\{\lambda=t e^{i \psi+\pi}\right.$ : $t>0\}$ of set $J(D)$. By means of a shift, a convex compact set can be put inside narrow domain $K$ if and only if the inequality

$$
H_{D}\left(e^{i \psi}\right)+H_{D}\left(e^{i \psi+\pi}\right)>H_{K}\left(e^{i \psi}\right)+H_{K}\left(e^{i \psi+\pi}\right)
$$

holds true.

If $D$ is a wide domain, then for each convex compact set $K$ there exists a shift which puts this set inside domain $D$. Thus, if the sequence $\Lambda=\left\{\lambda_{k}\right\}_{k=1}^{\infty}$ is so that $\bar{\tau}(\Lambda)<\infty$, then, as in Lemma 10 , system $\mathcal{E}=\left\{\exp \left(\lambda_{k} z\right)\right\}_{k=1}^{\infty}$ is incomplete in $H(D)$. 
Theorem 1. Suppose $\Lambda=\left\{\lambda_{k}\right\}_{k=1}^{\infty}$ is an unbounded strictly increasing sequence of positive numbers and $D$ is an unbounded convex domain in $\mathbb{C}$ such that the positive semi-axis lies on the boundary of set $J(D)$ but does not belong to it. Then the following statements are equivalent

1. Each function in $W(\Lambda, D)$ can be represented by series (1) in the half-plane $\left\{z \in \mathbb{C}: \operatorname{Re} z<H_{D}(1)\right\}$ (i.e., it is analytically continued into the half-plane and is represented there by the series);

2. $S_{\Lambda}=0$.

Proof. Suppose $z_{0} \in D$. By the hypothesis, the positive semi-axis lies on the boundary of set $J(D)$. Therefore, one of the rays $l_{1}=\left\{z=z_{0}+t e^{i \pi / 2}, t \geq 0\right\}, l_{2}=\left\{z=z_{0}+t e^{-i \pi / 2}, t \geq 0\right\}$ (possibly, both) lies in domain $D$. For clarity's sake, let it be ray $l_{1}$. We choose $\varepsilon_{0}>0$ such that the circle $B\left(z_{0}, 2 \varepsilon_{0}\right.$ lies inside $D$. Then domain $l_{1}+B\left(z_{0}, 2 \varepsilon_{0}\right)$ is contained in $D$ as well. By Lemma 9, there exists an entire function $f$ of exponential type vanishing at the points $\lambda_{k}$, $k \geq 1$, and a number $\gamma_{0}>0$ satisfying inequality (35) (as $\left.\tilde{\varepsilon}=\varepsilon_{0}\right)$. Let $K$ be the adjoint diagram for function $f$. Then according to (35), for all points $\lambda$ not lying on the real axis we have

$$
H_{K}(\lambda)=h_{f}(\lambda) \leqslant \pi|\operatorname{Im} \lambda| / \gamma_{0}+\varepsilon_{0}|\lambda| .
$$

Since the support function is continuous, this inequality can be continued on the whole plane. Let $t_{0}>\pi / \gamma_{0}$. Consider the function $f_{0}(\lambda)=f(\lambda) \exp \left(\lambda \tilde{z}_{0}\right)$, where $\tilde{z}_{0}=z_{0}+t_{0} e^{i \pi / 2}$. Its adjoint diagram is the compact set $K+\tilde{z}_{0}$ which, as it is easy to see, lies in domain $l_{1}+B\left(z_{0}, 2 \varepsilon_{0}\right)$ and thus in $D$. Hence, $f_{0}$ belongs to space $P_{D}$ and vanishes at the points $\lambda_{k}, k \geq 1$. It means that system $\mathcal{E}=\left\{\exp \left(\lambda_{k} z\right)\right\}_{k=1}^{\infty}$ is incomplete in $H(D)$.

Now we can apply Theorem 5.1 in work [3]. If Item 1 holds true, by this theorem we have the identity $S_{\Lambda}=0$ (under the hypothesis of the theorem the quantity $S_{\Lambda}(D)$ involved in the cited theorem coincides with $S_{\Lambda}$ ).

Let us prove the opposite. Suppose Item 2 holds true and let us show that in this case Item 5 in Theorem 5.1 of work [3] is valid. Let $L$ be an arbitrary convex set in domain $D$. Then $H_{D}(\lambda)>H_{L}(\lambda), \lambda \neq 0$. In accordance with the definition of the support function and the continuity of the support function of a compact set, we find a point $z_{1} \in D$ and numbers $\tilde{\varepsilon}, \tilde{\delta}>0$ satisfying inequality

$$
\operatorname{Re} z_{1}-\tilde{\varepsilon}>H_{L}(\lambda), \quad \lambda \in B(1, \tilde{\delta})
$$

Lessening $\tilde{\varepsilon}>0$, if necessary, we can assume that the circle $B\left(z_{1}, 2 \tilde{\varepsilon}\right)$ lies in domain $D$. Then $D$ contains also the domain $l_{1}+B\left(z_{1}, 2 \tilde{\varepsilon}\right)$. Let the a number $\gamma_{0} \in(0,1)$ and a function $f(\lambda)$ satisfy inequality (35). We indicate $f_{1}(\lambda)=f(\lambda) \exp \left(\lambda \tilde{z}_{1}\right)$, where $\tilde{z}_{1}=z_{1}+t_{1} e^{i \pi / 2}$ and $t_{1}>\pi / \gamma_{0}$. Function $f_{1}$ vanishes at the points $\lambda_{k}, k \geq 1$. Moreover, its adjoint diagram lies in domain $l_{1}+B\left(z_{1}, 2 \tilde{\varepsilon}\right)$, and this, in $D$, i.e., $f_{1} \in P_{D}$.

Suppose $\delta>0$. Let us show that for some $T(\delta)>0$ each point $\lambda_{k}$ obeying $\left|\lambda_{k}\right|>T(\delta)$ belongs to the set $R^{\delta}$, where $R=\left\{z: \ln \left|f_{1}(\lambda)\right| \geq H_{L}(\lambda)\right\}$.

We denote $T(\delta)=\max \left\{T, \gamma_{0} / \tilde{\delta}, \gamma_{0} / \delta\right\}$, where $T>0$ is the same as in (35). Let $\left|\lambda_{k}\right|>T(\delta)$. Then $\left|\lambda_{k}-i \gamma_{0}-\lambda_{k}\right| \leqslant \delta \lambda_{k}<\delta\left|\lambda_{k}-i \gamma_{0}\right|$ and by (35) we have

$$
\ln \left|f\left(\lambda_{k}-i \gamma_{0}\right)\right| \geq \frac{\left|\operatorname{Im}\left(\lambda_{k}-i \gamma_{0}\right)\right|}{\gamma_{0}}-\tilde{\varepsilon}\left|\lambda_{k}-i \gamma_{0}\right|=\pi-\tilde{\varepsilon}\left|\lambda_{k}-i \gamma_{0}\right| \geq-\tilde{\varepsilon} \lambda_{k} .
$$

By (50) and the homogeneity of the support function we obtain

$$
\begin{gathered}
\ln \left|f_{1}\left(\lambda_{k}-i \gamma_{0}\right)\right|=\ln \left|f\left(\lambda_{k}-i \gamma_{0}\right)\right|+\operatorname{Re}\left(\tilde{z}_{1}\left(\lambda_{k}-i \gamma_{0}\right)\right) \geq-\tilde{\varepsilon} \lambda_{k}+\lambda_{k} \operatorname{Re} \tilde{z}_{1}+\gamma_{0} \operatorname{Im} \tilde{z}_{1}= \\
=-\tilde{\varepsilon} \lambda_{k}+\lambda_{k} \operatorname{Re} z_{1}+\gamma_{0} \operatorname{Im} \tilde{z}_{1} \geq H_{L}\left(\lambda_{k}-i \gamma_{0}\right) .
\end{gathered}
$$

Here we suppose that $\operatorname{Im} \tilde{z}_{1} \geq 0$. If $\operatorname{Im} \tilde{z}_{1}<0$, then instead of $\lambda_{k}-i \gamma_{0}$, one should take $\lambda_{k}+i \gamma_{0}$.

We have shown that $\lambda_{k} \in R^{\delta}$, if $\left|\lambda_{k}\right|>T(\delta)$. Thus, the hypothesis of Theorem 5.1 in work [3] is satisfied and as well as Item 5 of this Theorem. This is why, Item 2 of this theorem holds true as well, and in accordance with it, each function in $W(\Lambda, D)$ is represented by series 
(1) in domain $D$. Then by Abel theorem for Dirichlet series (see, for instance, [1, Ch. II, Sect. 1, Subsect. 2,4]), each function in $W(\Lambda, D)$ is represented by series (1) in the half-plane $\left\{z \in \mathbb{C}: \operatorname{Re} z<H_{D}(1)\right\}$. The proof is complete.

We note that each half-plane $D=\{z: \operatorname{Re} z<a\}$ satisfies the hypothesis of the theorem. This is why the following statement holds true.

Corollary. Let $\Lambda=\left\{\lambda_{k}\right\}_{k=1}^{\infty}$ be an unbounded strictly increasing sequence of positive numbers and $D=\{z: \operatorname{Re} z<a\}$. Each function in $W(\Lambda, D)$ is represented by series (1) in half-plane $D$ if and only if $S_{\Lambda}=0$.

Theorem 2. Let $\Lambda=\left\{\lambda_{k}\right\}_{k=1}^{\infty}$ be an unbounded strictly increasing sequence of positive numbers and $D$ be an unbounded convex domain in $\mathbb{C}$ such that the positive semi-axis belongs to set $J(D)$. Then the following statements are equivalent

1. Each function in $W(\Lambda, D)$ is represented by series (1) in the whole plane;

2. $S_{\Lambda}>-\infty$.

Proof. As we have mentioned above, under the hypothesis of the theorem, system $\mathcal{E}$ is incomplete in $H(D)$. Then by Theorem 5.1 in work [3], Item 1 of the present theorem implies Item 2.

Let us prove the opposite and check Item 5 of Theorem 5.1 in work [3]. Inequality $S_{\Lambda}>-\infty$ holds due to Item 2. In our case, other statements of Item 5 of this theorem hold true trivially, since all the points $\lambda_{k}$ belong to the real semi-axis lying in $J(D)$. Thus, by Theorem 5.1 in work [3], each function in $W(\Lambda, D)$ is represented by series (1) in domain $D$. Since the support function of $D$ is unbounded on the real semi-axis, by Abel theorem for Dirichlet series, each of such series converges in the whole plane. The proof is complete.

In the particular case $D=\mathbb{C}$ we obtain

Corollary. Let $\Lambda=\left\{\lambda_{k}\right\}_{k=1}^{\infty}$ be an unbounded strictly increasing sequence of positive numbers. There following statements are equivalent

1. Each function in $W(\Lambda, \mathbb{C})$ is represented by series (1) in the whole plane;

2. $S_{\Lambda}>-\infty$;

3. Each function in $W(\Lambda, \mathbb{C})$ is represented by series (1) on some open subset of the plane.

Proof. Items 1 and 2 are equivalent by Theorem 2. The implication Item $1 \Rightarrow$ Item 3 is trivial. The implication Item $3 \Rightarrow$ Item 2 follows from Lemma 3 . The proof is complete.

Theorem 3. Let $\Lambda=\left\{\lambda_{k}\right\}_{k=1}^{\infty}$ be an unbounded strictly increasing sequence of positive numbers, $D$ is an unbounded convex domain in $\mathbb{C}$ such that the real semi-axis belongs to set $J(D)$ and $\psi \in[0, \pi)$ is so that $H_{D}\left(e^{i \psi}\right)<+\infty$ and $H_{D}\left(e^{i \psi+\pi}\right)<+\infty$. Then the following statements are equivalent

1. Each function in $W(\Lambda, D)$ is represented by series (1) in the whole plane;

2. $S_{\Lambda}>-\infty$ and

$$
|\sin \psi| \lim _{a \rightarrow+\infty} \frac{1}{\ln a} \varlimsup_{r \rightarrow+\infty} \sum_{r \leqslant \lambda_{k}<a r} \operatorname{Re} \frac{1}{\lambda_{k}}<\frac{1}{2 \pi}\left(H_{D}\left(e^{i \psi}\right)+H_{D}\left(e^{i \psi+\pi}\right)\right) .
$$

Proof. If Item 1 holds, then by Lemma 10, system $\mathcal{E}$ is incomplete in $H(D)$. Then, as one easily see, Theorem 2 in work [14] yields (51). Applying then Theorem 5.1 in work [3], we obtain Item 2.

And vice versa, if (51) holds, by Theorem 2 in work [14], system $\mathcal{E}$ is incomplete in $H(D)$. Then by Theorem 5.1 in work [3], the implication Item $2 \Rightarrow$ Item 1 holds true. The proof is complete.

Theorem 4. Let $\Lambda=\left\{\lambda_{k}\right\}_{k=1}^{\infty}$ be an unbounded strictly increasing sequence of positive numbers, $D$ be a convex domain in $\mathbb{C}$ such that function $H_{D}(\lambda)$ is bounded in the vicinity of $\lambda=1$. Then the following statements are equivalent 
1. Each function in $W(\Lambda, D)$ can be represented by series (1) in the half-plane $\left\{z \in \mathbb{C}: \operatorname{Re} z<H_{D}(1)\right\}$

2. System $\mathcal{E}$ is incomplete in $H(D), S_{\Lambda}=0, \tau_{0}(\Lambda)<+\infty$, and the intersection of the support line $\left\{z \in \mathbb{C}: \operatorname{Re} z=H_{D}(1)\right\}$ with the boundary of the domain $D$ contains the segment of length $2 \pi \tau_{0}(\Lambda)$.

Proof. Suppose Item 2 holds true. Let us show that the hypothesis of Theorem 3.6 in work [3] holds true. Conditions 1 and 2 are equivalent, i.e., $S_{\Lambda}=0$. Conditions 3 and 4 are trivially satisfied since all the points $\lambda_{k}$ belong to the positive real semi-axis, in the vicinity of which the support function of domain $D$ is bounded. It remains to check Condition 5 . Such check has already been made in Lemma 7. In accordance with this lemma, condition 5 of Theorem 3.6 in work [3] is also satisfied. Then by this theorem and Proposition 2.10 of work [3], each function $W(\Lambda, D)$ is represented by series (1) in domain $D$. As above, by Abel theorem for Dirichlet series, we obtain Item 1 of the present theorem.

Suppose Item 1 holds true and let $\tilde{S}_{\Lambda}=-\infty$. Then by Lemma 4 , for each $\tau>0$, there exist $\delta>0$ and function $g_{\tau} \in W(\Lambda, G)$, where $G=(\{z: \operatorname{Re} z<\tau \delta\} \cap B(0, \tau)) \cup\{z: \operatorname{Re} z<0\}$ represented by series (1) with the convergence abscissa $\gamma=0$. We let $X=\partial D \cap\{z: \operatorname{Re} z=$ $\left.H_{D}(1)\right\}$. By assumption, $H_{D}(\lambda)$ is bounded in the vicinity of $\lambda=1$. This is why $X$ is either a point or a segment. If $X$ consists of the only point, we denote it by $z_{0}$ otherwise this symbols stands for the center of segment $X$. Let $\tau>0$ be strictly greater than the length of $X$ (possibly being zero). Then for some $\tilde{\delta} \in(0, \delta)$, the domain $\tilde{G}=G+z_{0}-\tilde{\delta}$ contains $D$. We let $g(z)=g_{\tau}\left(z-z_{0}+\tilde{\delta}\right)$. Function $g$ belongs to space the $W(\Lambda, \tilde{G}) \subset W(\Lambda, D)$ and is represented by series (1) with convergence abscissa $\gamma=\operatorname{Re} z_{0}-\tilde{\delta}=H_{D}(1)-\tilde{\delta}$. Since the expansion into the Dirichlet series is unique, it contradicts Item 1.

Thus, $\tilde{S}_{\Lambda}>-\infty$. As it has already been mentioned, it yields $S_{\Lambda}=0$. Moreover, by Lemma 1 , the maximal density $\tau_{0}(\Lambda)$ is finite and by Lemma 10-, system $\mathcal{E}$ is incomplete in $H(D)$. It remains to show that the length of set $X$ is at least $2 \pi \tau_{0}(\Lambda)$.

Suppose the length $X$ equals $2 \pi \tau^{\prime}<2 \pi \tau_{0}(\Lambda)$. We choose $\alpha>0$ so that $\tau^{\prime}+\alpha<\tau_{0}(\Lambda)$. Consider the domains

$$
\begin{aligned}
& D^{\prime \prime}=\left\{z: \operatorname{Re} z<H_{D}(1)\right\} \cap\left\{z: \operatorname{Re}\left(z e^{-i \beta}\right)<\operatorname{Re}\left(\left(z_{0}+i\left(\tau^{\prime}+\alpha\right)\right) e^{-i \beta}\right)\right\} \cap \\
& \left.\cap\left\{z: \operatorname{Re}\left(z e^{i \beta}\right)\right)<\operatorname{Re}\left(\left(z_{0}-i\left(\tau^{\prime}+\alpha\right)\right) e^{i \beta}\right)\right\}, \quad D^{\prime}=D^{\prime \prime} \cap\{z: \operatorname{Re} z>b\} .
\end{aligned}
$$

It is easy to see that domain $D^{\prime \prime}$ is unbounded and convex and it contains $D$ for some $\beta>0$, and domain $D^{\prime} \subset D^{\prime \prime}$ is an isosceles trapezium (as $b<H_{D}(1)$ ) whose bases are parallel to the imaginary axis. One of them lies on the support line $\left\{z: \operatorname{Re} z=H_{D}(1)\right\}$ of domain $D$, contains $X$, and has the length $2 \pi\left(\tau^{\prime}+\alpha\right)$. The length of the other is strictly greater $2 \pi\left(\tau^{\prime}+\alpha\right)$, and it increases as $b$ decreases. We choose $b \in \mathbb{R}$ such that this length becomes strictly greater than $2 \pi \tau_{0}(\Lambda)$. Then domain $D^{\prime}$ contains some shift of the segment $\left[-i \pi \tau_{0}(\Lambda), i \pi \tau_{0}(\Lambda)\right]$. Let $f$ be a function whose existence is stated in Lemma 6 . Since $f$ vanishes at points $\lambda_{k}, k \geq 1$, system $\mathcal{E}$ is incomplete in $H\left(D^{\prime}\right)$. This is why space $W\left(\Lambda, D^{\prime}\right)$ is non-trivial.

Suppose each function in $W\left(\Lambda, D^{\prime}\right)$ is represented by series (1) in domain $D^{\prime}$. Since it is bounded, by Theorem 5.2 in work [3], there exists an entire function $\varphi$ of exponential type vanishing at points $\lambda_{k}, k \geq 1$, having regular growth everywhere in the plane, and its adjoint diagram coincides with the closure of domain $D^{\prime}$. Then by (2), the identity

$$
\tau(-\varphi, \varphi, \tilde{\Lambda})=\frac{1}{2 \pi} s\left(-\varphi, \varphi, D^{\prime}\right)
$$

holds true except at most a countable set of values of $\varphi$. Here $\tilde{\Lambda}$ is the zero set of $f, s\left(-\varphi, \varphi, D^{\prime}\right)$ is the length of the arc $\gamma(\varphi)$ on boundary $\partial D^{\prime}$ connecting the support points $z(\varphi), z(-\varphi) \in \partial D^{\prime}$ of the support lines $l( \pm \varphi)=\left\{z: \operatorname{Re}\left(z e^{ \pm \varphi i}\right)=H_{D}^{\prime}\left(e^{ \pm \varphi i}\right)\right\}$, respectively. Since $\Lambda$ is a part of a 
measurable set $\tilde{\Lambda}$, we have

$$
\begin{aligned}
\tau_{0}(\Lambda)=\varlimsup_{\delta \rightarrow 0} \varlimsup_{r \rightarrow \infty} & \frac{n(r, \Lambda)-n((1-\delta) r, \Lambda)}{\delta r} \leqslant \varlimsup_{\delta \rightarrow 0} \varlimsup_{r \rightarrow \infty} \frac{n(-\varphi, \varphi, r, \Lambda)-n(-\varphi, \varphi,(1-\delta) r, \Lambda)}{\delta r}= \\
& =\varlimsup_{\delta \rightarrow 0}\left(\varlimsup_{r \rightarrow \infty} \frac{n(-\varphi, \varphi, r, \Lambda)}{\delta r}-\varlimsup_{r \rightarrow \infty} \frac{n(-\varphi, \varphi,(1-\delta) r, \Lambda)}{\delta r}\right)= \\
& =\varlimsup_{\delta \rightarrow 0}\left(\frac{\tau(-\varphi, \varphi, \tilde{\Lambda})}{\delta}-\frac{(1-\delta) \tau(-\varphi, \varphi, \tilde{\Lambda})}{\delta}\right)=\tau(-\varphi, \varphi, \Lambda)
\end{aligned}
$$

except at most a countable set of values of $\varphi$. As $\varphi$ is small enough, the arc $\gamma(\varphi)$ coincides with the basis of trapezium $D^{\prime}$ of length $2 \pi\left(\tau^{\prime}+\alpha\right)$. This is why by (52), inequality $\tau_{0}(\Lambda) \leqslant \tau^{\prime}+\alpha$ should hold that contradicts the choice of number $\alpha$.

Hence, there exists a function $g^{\prime} \in W\left(\Lambda, D^{\prime}\right)$ which can not be represented by (1) in domain $D^{\prime}$. Now consider the domain $\tilde{D}^{\prime}=D^{\prime} \cap\{z: \operatorname{Re} z<a\}$, where $b<a<H_{D}$. It lies in $D^{\prime}$ and is an isosceles trapezium whose bases are parallel to the imaginary axis. One of them coincides with that of $D^{\prime}$ having the length strictly greater than $2 \pi \tau_{0}(\Lambda)$. We choose a number $a$ such that the other basis of $\tilde{D}^{\prime}$ has a length strictly greater than $2 \pi \tau_{0}(\Lambda)$. System $\mathcal{E}$ is incomplete in $H\left(\tilde{D}^{\prime}\right)$ due to the same reasons as in $H\left(D^{\prime}\right)$. Then by the proven implication Item $2 \Rightarrow$ Item 1 , function $g^{\prime} \in W\left(\Lambda, \tilde{D}^{\prime}\right)$ is represented by series (1) in the half-plane $\{z: \operatorname{Re} z<a\}$. The union of the latter and domain $D^{\prime}$ contains $D^{\prime \prime}$. This is why $g^{\prime} \in W\left(\Lambda, \tilde{D}^{\prime}\right) \cap H\left(D^{\prime \prime}\right)$. Since domain $D^{\prime \prime}$ is unbounded, by Theorem 8.1 in work [15], the inclusion $g^{\prime} \in W\left(\Lambda, D^{\prime \prime}\right)$ holds true as well. Domain $D$ lies in $D^{\prime \prime}$. Therefore, $g^{\prime} \in W(\Lambda, D)$. Due to Item 1 , function $g^{\prime}$ is represented by series (1) in the half-plane $\left\{z \in \mathbb{C}: \operatorname{Re} z<H_{D}(1)\right\}$ containing $D^{\prime}$. It contradicts to the choice of $g^{\prime}$.

Thus, the length of segment $X$ is at least $2 \pi \tau_{0}(\Lambda)$. The proof is complete.

Remark. If sequence $\Lambda$ has the density $\tau(\Lambda)$ (and then $\tau(\Lambda)=\tau_{0}(\Lambda)$ ), system $\mathcal{E}$ is incomplete in $H(D)$ if and only domain $D$ contains some vertical segment of length $2 \pi \tau_{0}(\Lambda)$ (see [16, Ch. III, Sect. 1, Thm. 3.1.6]). If domain $D$ is unbounded, then, as above, the condition of incompleteness for system $\mathcal{E}$ is incomplete in $H(D)$ can be either excluded or replaced by (51).

Corollary 1. Let $\Lambda=\left\{\lambda_{k}\right\}_{k=1}^{\infty}$ be an unbounded strictly increasing sequence of positive numbers, $D$ be an unbounded wide convex domain in $\mathbb{C}$ such that function $H_{D}(\lambda)$ is bounded in the vicinity of $\lambda=1$. Then the following statements are equivalent

1. Each function in $W(\Lambda, D)$ can be represented by series (1) in the half-plane $\left\{z \in \mathbb{C}: \operatorname{Re} z<H_{D}(1)\right\}$

2. $S_{\Lambda}=0, \tau_{0}(\Lambda)<+\infty$ and the intersection of the support line $\left\{z \in \mathbb{C}: \operatorname{Re} z=H_{D}(1)\right\}$ with the boundary of domain $D$ contains the segment of length $2 \pi \tau_{0}(\Lambda)$.

Corollary 2. Let $\Lambda=\left\{\lambda_{k}\right\}_{k=1}^{\infty}$ be an unbounded strictly increasing sequence of positive numbers, $D$ be an unbounded convex domain in $\mathbb{C}$ such that function $H_{D}(\lambda)$ is bounded in the vicinity of $\lambda=1$ and $\psi \in[0, \pi)$ is so that $H_{D}\left(e^{i \psi}\right)<+\infty$ and $H_{D}\left(e^{i \psi+\pi}\right)<+\infty$. Then the following statements are equivalent

1. Each function in $W(\Lambda, D)$ can represented by series (1) in the half-plane $\left\{z \in \mathbb{C}: \operatorname{Re} z<H_{D}(1)\right\}$;

2. There holds (51), $S_{\Lambda}=0, \tau_{0}(\Lambda)<+\infty$, and the intersection of the support line $\left\{z \in \mathbb{C}: \operatorname{Re} z=H_{D}(1)\right\}$ and of the boundary of domain $D$ contains a segment of length $2 \pi \tau_{0}(\Lambda)$.

\section{BIBLIOGRAPHY}

1. A.F. Leont'ev. Exponentials series. Nauka, Moscow. 1976. (in Russian).

2. A.A. Gol'dberg, B.Ya. Levin, I.V. Ostrovskii Entire and meromorphic functions // Itogi nauki i tekhniki. Sovremen. problemy matem. Fundament. napravl. VINITI, Moscow. 1991. P. 5-185. (in Russian). 
3. A.S. Krivosheev. A fundamental principle for invariant subspaces in convex domains // Izv. RAN. Ser. matem. 2004. V. 68, No. 2. P. 71-136. [Izv. Math. 2004. V. 68, No. 2. P. 291-353.]

4. O.A. Krivosheeva, A.S. Krivosheev. A criterion for the fundamental principle to hold for invariant subspaces on bounded convex domains in the complex plane // Funkts. analiz. 2012. V. 46, No. 4. P. 14-30. [Funct. Anal. Appl. 2012. V. 46, No. 4. P. 249-261.]

5. B.Ya. Levin. Distribution of zeros of entire functions. Gostekhizdat, Moscow. 1956. [Amer. Math. Soc., Providence, RI. 1980.]

6. P. Lelong, L. Gruman. Entire functions of several complex variables. Springer-Verlag, Berlin. 1986.

7. A.F. Leont'ev. Entire functions. Exponential series. Nauka, Moscow. 1983.

8. V.V. Napalkov. Convolution equations in multidimensional spaces. Nauka, Moscow, 1982.

9. P. Koosis. The logarithmic integral I. Cambridge University Press, 1997.

10. O.A. Krivosheyeva. Singular points of the sum of a series of exponential monomials on the boundary of the convergence domain // Algebra i analiz. 2011. V. 23, No. 2. P. 162-205. [St. Petersburg Math. J. 2012. V. 23, No. 2. P. 321-350.]

11. O.A. Krivosheyeva. The convergence domain for series of exponential monomials // Ufimskij matem. zhur. 2011. V. 3, No. 2. P. 43-56. [Ufa Math. J. 2011. V. 3, No. 2. P. 42-55.]

12. R.S. Yulmukhametov. Approximation of subharmonic functions // Analysis Mathematica. 1985. V. 11, No. 3. P. 257-282.

13. I.F. Krasichkov. Comparison of entire functions of finite order by means of the distribution of their roots // Matem. sbornik. 1966. V. 70(112), No. 2. P. 198-231. (in Russian).

14. B.N. Khabiullin. On the growth of entire functions of exponential type along the imaginary axis // Matem. sbornik. 1989. V. 180, No. 5. P. 706-719. [Math USSR Sb. 1989. V. 67, No. 1. P. 149-163.]

15. I.F. Krasičkov-Ternovskii. Invariant subspaces of analytic functions. II. Spectral synthesis of convex domains // Matem. sbornik. 1972. V. 88(130), No. 1. P. 3-30. [Math USSR Sb. 1972, V. 17, No. 1. P. 1-29.]

16. A.F. Leont'ev. Sequence of exponential polynomials. Nauka, Moscow. 1980. (in Russian).

Alexandr Sergeevich Krivosheev,

Institute of Mathematics USC RAS,

Chernyshevsky str., 112,

450008, Ufa, Russia

Olesya Alexandrovna Krivosheyeva,

Bashkir State University,

Z. Validi str., 32,

450074, Ufa, Russia

E-mail: kriolesya2006@yandex.ru 